\title{
Requirement of Irf6 and Esrp1/2 in frontonasal and palatal epithelium to regulate craniofacial and palate morphogenesis in mouse and zebrafish
}

Shannon H. Carroll ${ }^{1,2, \#}$, Claudio Macias Trevino ${ }^{1,3, \#}$, Edward B-H Li ${ }^{3}$, Kenta Kawasaki ${ }^{1,2}$, Nora Alhazmi $^{4}$, Shawn Hallett ${ }^{1}$, Justin Cotney ${ }^{7}$, Russ P. Carstens ${ }^{6}$ and Eric C. Liao ${ }^{1,2,3,{ }^{*}}$

${ }^{1}$ Center for Regenerative Medicine, Massachusetts General Hospital, Boston, MA 02114, USA. ${ }^{2}$ Shriners Hospital for Children, Boston, MA 02114, USA.

${ }^{3}$ Harvard Medical School, Boston, MA 02115, USA.

${ }^{4}$ Harvard School of Dental Medicine, Boston, MA 02115, USA.

${ }^{5}$ Division of Plastic and Reconstructive Surgery, Massachusetts General Hospital, Boston, MA 02114, USA.

${ }^{6}$ Department of Medicine, Perelman School of Medicine, University of Pennsylvania, Philadelphia, PA 19104, USA.

${ }^{7}$ Department of Genetics and Genome Sciences, University of Connecticut Health, CT 06030, USA.

\#Authors contributed equally to this work

*Author for correspondence: cliao@partners.org

Telephone: ECL: (617) 643-5975

Key words: IRF6, ESRP1, craniofacial, cleft, development 


\section{SUMMARY STATEMENT}

These studies elucidate Irf6 and Esrp1/2 function in periderm and embryonic epithelium during palate development, using complementary mouse and zebrafish models. Mouse and zebrafish Irf6 and Esrp1/2 develop cleft lip and palate, where an aberrant mesenchymal/epithelial cell population is localized to the cleft in the esrp1/2 zebrafish mutant.

\section{ABSTRACT}

Orofacial clefts are among the most common human congenital malformations. Irf6 and Esrp1 are two key genes important for palate development, conserved across vertebrates. In the zebrafish, we found that irf6 regulates the expression of esrp1. Using RNAscope, we detailed overlapping Irf6 and Esrp1/2 gene expression in the mouse frontonasal prominence ectoderm, lambda joint periderm, palate and lip epithelium. In the zebrafish, irf6 and esrp1/2 share expression in the pre-gastrulation periderm and the embryonic frontonasal ectoderm, oral epithelium ventral to the anterior neurocranium (ANC), and the developing stomodeum. Genetic disruption of irf6 and esrp1/2 in the zebrafish resulted in cleft of the ANC. In the esrp1/2 zebrafish mutant, cleft of the mouth opening formed and appeared to tether into the ANC cleft. Lineage tracing of the anterior cranial neural crest cells revealed that cleft of the ANC resulted not from migration defect, but from impaired chondrogenesis. Molecular analysis of the aberrant cells localized within the ANC cleft revealed that this cell population espresses sox10, col1a1 and irf6 and is adjacent to cells expressing epithelial krt4. Detailed morphogenetic analysis of mouse Irf6 mutant revealed mesenchymal defects not observed in the Esrp1/2 mutant. Analysis of breeding compound Irf6;Esrp1;Esrp2 mutant suggests that these genes interact where the triple mutant is not observed. Taken together, these studies 
highlight the complementary analysis of Irf6 and Esrp1/2 in mouse and zebrafish models and captured an unique aberrant embryonic cell population that contributes to cleft pathogenesis. Future work characterizing this unqiue sox10+, col1a1+, irf6+ cell population will yield additional insight into cleft pathogenesis. 


\section{INTRODUCTION}

The development of vertebrate craniofacial structures requires coordinated cellular induction, migration, proliferation, and differentiation, which allow for the positioning of adjacent epitheliallined facial processes that ultimately merge(Abramyan and Richman, 2015; Cordero et al., 2011; Creuzet et al., 2005; Dougherty et al., 2012; Helms et al., 2005; Jiang et al., 2006; Knight and Schilling, 2006; Mork and Crump, 2015; Reid et al., 2011). Morphogenesis of facial structures such as the midface, lip, and palate requires convergence of the medial and lateral nasal prominences and the fusion of the secondary palatal shelves at the midline (Abramyan and Richman, 2015; Jiang et al., 2006; Losa et al., 2018). Failure of these processes to fuse results in orofacial clefts (OFCs) of the lip, primary palate, or secondary palate (Gritli-Linde, 2008). Orofacial clefts are among the most common congenital structural anomalies (Goodwin et al., 2015; Juriloff and Harris, 2008; Yuan et al., 2011). From genome-wide association studies over a decade ago to more recent whole-genome sequencing projects of orofacial cleft cohorts, human geneticists have identified several cleft-associated genetic loci, where the transcription factor IRF6 continues to be one of the most commonly associated genes (Marazita, 2012; Zucchero et al., 2004). IRF6 disruption is causal for syndromic cleft in Van der Woude and Popliteal Pterygium syndromes, and associated with non-syndromic orofacial clefts (Beaty et al., 2016; Kondo et al., 2002; Leslie et al., 2013; Zucchero et al., 2004).

Several IRF6 transcriptional targets such as GRHL3, WDR65, OVOL1, KLF4 have been identified, which are also important for palate development and implicated in human cleft pathogenesis (de la Garza et al., 2013; Kousa and Schutte, 2016; Liu et al., 2016; Rorick et al., 2011). These studies support the premise that investigation of Irf6 and its transcriptional 
network will identify key genes that regulate palate development. Multiple mouse models have been generated to investigate Irf6 function, including a total Irf6 knockout as well as amino acid substitution of key functional residue $\operatorname{Irf} 6^{R 84 C}$ in the DNA-binding domain (Ingraham et al., 2006; Richardson et al., 2006). These Irf6 mutant mice exhibited disrupted epithelial terminal differentiation and lack of a functional periderm, leading to pathological adhesions of epithelial tissues (Ferretti et al., 2011; Ingraham et al., 2006; Iwata et al., 2013; Richardson et al., 2006). The epithelial differentiation and adhesion defects are thought to prevent elevation of the palatal shelves, and ultimately these mice have a cleft in the secondary palate. Additionally, the midface of these mice were hypoplastic, which was attributed to a dysfunctional epithelium (Ingraham et al., 2006; Richardson et al., 2006).

Epithelial splicing regulatory factors 1 and 2 (Esrp1, Esrp2) are also important in embryonic epithelial differentiation and palate development (Bebee et al., 2015; Lee et al., 2018; Lee et al., 2020). Esrp2 and its homolog Esrp1 are regulators of RNA splicing that are specifically expressed in the epithelium (Warzecha et al., 2009). Esrp1/2 knockout mice exhibit bilateral cleft of the lip and primary palate, as well as a secondary palate cleft (Bebee et al., 2015). Esrp1/2 are unusual among regulators of RNA-splicing in that they are tissue-restricted and exhibit dynamic expression during embryogenesis (Bebee et al., 2015; Burguera et al., 2017). The developmental importance of Esrp1/2 is underscored by their conservation across species, from ascidians to zebrafish, Xenopus, mouse, and humans (Burguera et al., 2017). Gene variant in ESRP2 was also recently reported in human orofacial cleft cohorts (Cox et al., 2018). 
The mouse has been an important experimental model to study craniofacial and palate development (Gritli-Linde, 2008). Secondary palate development in the mouse is similar to humans, with the analogous stages of vertical outgrowth, elevation, horizontal growth, and fusion (Gritli-Linde, 2008; Juriloff and Harris, 2008). Many genes associated with cleft lip and palate $(C L / P)$ in humans when disrupted in the mouse result in cleft of the secondary palate, but the primary palate and lip appear unaffected (Gritli-Linde, 2008; Van Otterloo et al., 2016). So while the mouse model can be useful to study the secondary palate, the use of mouse models to study cleft of the lip and primary palate has been less effective as there are remarkably few mouse models where development of the lip and primary palate are perturbed (Gritli-Linde, 2008). Meanwhile, clinically CL/P is more common than isolated cleft of the palate only (CPO), and human genetic studies have suggested that the genetics underpinning CL/P and CPO are distinct (Gritli-Linde, 2008; Juriloff and Harris, 2008) The developmental processes of outgrowth of the facial prominences followed by convergence and fusion are thought to be conserved across mammals (Abramyan and Richman, 2015). Therefore it is hypothesized that differences in mouse versus human phenotypic presentation are due to spatiotemporal differences in craniofacial development (Gritli-Linde, 2008). In this context, the phenotype of bilateral clefts affecting the lip, primary and secondary palate in the Esrp1/2 mutant mouse is unique among mouse models and is a valuable tool to study lip and palate morphogenesis.

Zebrafish has been favored as an animal model by embryologists to study craniofacial development due to its accessibility and transparency (Kimmel, 1989; Lieschke and Currie, 2007; Schilling and Le Pabic, 2009). Although a secondary palate, which partially or entirely 
separates the oral and nasal cavities, is reserved to select amniotes, the primary palate is appreciably conserved across all vertebrates (Abramyan and Richman, 2015). The primary palate establishes the intact upper jaw (Abramyan and Richman, 2015), which in the larval zebrafish consists of the ethmoid plate, also known as the anterior neurocranium (ANC). In all vertebrates, the most anterior CNCCs that migrate rostral then turn caudal and ventral to the eye contribute to the median frontonasal prominence, and a second CNCC stream that migrates or inferior to the eye and into the first pharyngeal arch to generate the paired maxillary prominences (Dougherty et al., 2012; Kimmel, 1989; Swartz et al., 2011; Wada et al., 2005). The ANC of the zebrafish is formed from the convergence of the median element that is derived from the frontonasal prominence, and paired lateral elements that are derived from the maxillary prominences (Duncan et al., 2017; Mork and Crump, 2015; Swartz et al., 2011). Zebrafish homologs of human genes associated with orofacial clefts will disrupt morphology of the ANC, as have been observed fro a number of genes such as capzb, pitx2, pdgfra, smad5, tgfb2, fgf10a and wnt9a (Duncan et al., 2017; Mork and Crump, 2015; Van Otterloo et al., 2016)

Here, we carried out detailed gene expression analysis of Irf6, Esrp1 and Esrp2 in mouse and zebrafish in order to understand the comparative morphogenesis of facial structures and periderm between these common vertebrate genetic models. We analyzed and compared the Irf6 and Esrp1/2 mutant phenotypes to elucidate the comparative morphologies and genetic epistasis between these genes. Further, we generated zebrafish irf6 and esrp1/2 zebrafish mutants and examined their requirement in morphology of the stomodeum opening and ANC. Interestingly, we identified an aberrant cell population with epithelial and mesenchymal 
molecular signatures that localized to the region of the ANC cleft. This work highlights the relative strengths of the mouse and zebrafish models for investigating the morphogenetic mechanisms of orofacial clefts and contributes new insights into the function of Irf6 and Esrp1/2 during palatogenesis. 


\section{RESULTS}

\section{irf6 null zebrafish embryos have decreased expression of esrp1}

We previously reported generation of a functionally null irf6 zebrafish allele (Li et al., 2017). Using CRISPR/Cas9, an 8 bp deletion in exon 6 of the irf6 coding region resulting in a frameshift and premature stop codon, leading to the ablation of irf6 function. It was observed that embryos lacking maternally expressed irf6 exhibited epiboly arrest and periderm rupture at 4-5 hpf (Li et al., 2017). Utilizing this maternal irf6-null model, we aimed to identify genes that were differentially downregulated in irf6-null versus wild type embryos. We performed RNAseq on wild type and maternal/zygotic irf6-null (mz-irf6 $6^{8 \mathrm{bp} /-8 \mathrm{bp}}$ ) embryos at $4.5 \mathrm{hpf}$, just before embryo rupture at the onset of gastrulation, as irf6 is required for periderm integrity. Analysis of the RNA-seq identified differentially-expressed genes (DEGs) in wild type relative to mutant (Fig. 1A-C). The RNA-seq results revealed significant downregulation of genes previously known to be downregulated with disruptions in Irf6 function (Fig.1B,C). Disruption of irf6 via injecting dominant-negative irf6 mRNA led to downregulation of many periderm-enriched genes (including grhl1, krt5, krt18, tfap2a and klf2b) and genes for adhesion molecules (including claudins and cadherins) (de la Garza et al., 2013). Here we found a similar expression profile in the mz-irf6 ${ }^{-8 b p /-8 b p}$ embryos relative to wild type (Fig. 1B,C).

When compared to previously published IRF6 siRNA human keratinocyte DEG expression data (Botti et al., 2011), there were major overlaps of genes in molecular pathways responsible for epithelial regulation, including gata3, krt18 and cldn4 (Fig.1 B,C and Fig. S1).

In addition to the epithelial differentiation and maturation genes, many key developmental signaling pathways including Fgf (fgf8a, fgf17 and fgf24) and Wnt (wnt11, dact2, rspo3, frzb, $f z d 5)$ pathways were also heavily represented in our dataset as genes downregulated due to 
irf6 ablation (Fig. 1B,C). Further, a number of genes associated with human orofacial clefts (OFCs) are also downregulated in the irf6 null embryos, including hey1, gata3, wnt11, and fgf8 (Fig. 1B,C).

Interestingly, one of the most downregulated genes in the mz-irf6 ${ }^{-8 \mathrm{bp} /-8 \mathrm{bp}}$ embryos was esrp1. Epithelial splicing regulatory protein 1 (esrp1) and its paralog esrp2 are epithelial restricted RNA splicing regulators. ESRP2 genetic variants in humans are associated with OFCs (Cox et al., 2018), and Esrp1 and Esrp1/2 knockout mice display a bilateral cleft of the lip, primary and secondary palate (Bebee et al., 2015). To confirm the RNA-seq results, we performed qPCR on mz-irf6 ${ }^{8 \mathrm{bp} /-8 \mathrm{bp}}$ and wild type embryos at 4-5 hpf. Relative to wild type, mz-irf6-8bp/-8bp embryos had approximately 5-fold downregulation of esrp1 expression. Additionally, injection of mz-irf6 ${ }^{8 \mathrm{bp} /-8 \mathrm{bp}}$ embryos with irf6 mRNA at the 1-cell stage rescued esrp1 expression, resulting in increase that was approximately 3-fold higher than wild type (Fig. 1D). Therefore esrp1 gene expression is dependent on irf6, either through direct regulation or the requirement of a normal periderm.

\section{irf6, esrp1 and esrp2 are co-expressed in the oral epithelium of zebrafish during craniofacial development}

Previous mouse studies have described Irf6, Esrp1, and Esrp2 gene expression in epithelial cells during palate development (Bebee et al., 2015; Knight et al., 2006). To determine the gene expression of irf6 and esrp1 and esrp2 in the zebrafish during epithelial and craniofacial development, we performed whole-mount in situ hybridization (WISH) as well as RNAscope in situ hybridization (ISH) at 8-cell stage, shield stage, 48 and $72 \mathrm{hpf}$. WISH of zebrafish embryos demonstrated maternal deposition of irf6, esrp1 and esrp2 mRNA detectable at 8-cell 
stage (Fig. 2A). The maternal transcriopt of these genes are also detected in the periderm of the gastrulating embryo, although expression of esrp2 appears lower than irf6 and esrp1

(Fig.2A). During craniofacial development WISH demonstrated specific expression of irf6, esrp1, and esrp2 lining the embryonic oral epithelium, and circumscribing surface epithelium concentrated around the developing stomodeum (Fig. 2B,C). Gene expression domains of irf6, esrp1, and esrp2 overlap significantly, consistent with previous reports (Burguera et al., 2017).

To resolve the specific cell population of the embryonic epithelium that express irf6, esrp1, and esrp2, we performed RNAscope in-situ hybridization of coronal cryosections taken through the developing mouth and palate at 48 and 72 hours post-fertilization (hpf). We find that irf6 and esrp1 are co-expressed within epithelial cells lining the oral cavity as well as the surface epithelium (Fig. 2D,E). No expression of these genes was detected within the cartilage elements, identified by sox 10 expression. Further, we detected irf6 and esrp1 transcripts within the same cells, importantly within epithelial cells separating adjacent cartilage elements prior to the fusion of paired Meckel's cartilage elements derived from the mandibular facial prominence (Fig. 2G').

\section{Irf6, Esrp1, and Esrp2 are co-expressed in murine frontonasal and oral epithelium, during palate and lip development}

Irf6, Esrp1, and Esrp2 are each expressed within the embryonic oral epithelium surrounding the developing palatal shelves of mice (Bebee et al., 2015; Knight et al., 2006). Ablation of Irf6 or Esrp1/2 causes a cleft of the secondary palate, but the disruption of the lip and primary palate phenotypes differ between the Irf6 and Esrp1 and Esrp2 mutants (Bebee et al., 2015; 
Ingraham et al., 2006; Richardson et al., 2006). To determine whether Irf6, Esrp1 and Esrp2 transcripts co-localize during mouse craniofacial development similarly to zebrafish, we performed whole-mount ISH for each gene starting at E10.5 as the frontonasal prominences and lambdoidal junction are taking shape at this time point. We found that Irf6, Esrp1, and Esrp2 genes are expressed similarly in E10.5 embryos with high levels of expression in areas of craniofacial development (Fig. 3A). The mouse gene expression pattern was similar to that observed in zebrafish, with more concentrated expression to the developing head. Higherresolution imaging with RNAscope ISH to determine cellular co-localization detected Irf6, Esrp1, and Esrp2 transcripts in the periderm and the basal epithelium across all time points examined (Fig. 3B-F). Irf6, Esrp1 and Esrp2 are co-expressed in the surface ectoderm overlying the developing frontonasal prominences (Fig. 3B), a cell population with important signaling and inductive functions (Hu et al., 2003). Further, co-expression included cells at critical fusion points, specifically between the medial and lateral nasal prominences (Fig.3C) and the palatal shelves (Fig. 3E,F). Additionally, with RNAscope, we were able to resolve differences in the mRNA expression pattern of Esrp1 and Esrp2, with Esrp2 generally being more highly expressed in the apical epithelial layer. In contrast, Esrp1 is evenly expressed throughout the apical and basal epithelial layer. Interestingly, in addition to Irf6 expression in the epithelium, RNAscope detected Irf6 mRNA expression in the mesenchyme, particularly at E10 and E15.5 (Fig. 3B,E). Expression of Irf6 in this craniofacial mesenchyme has not been reported. However, expression was detected in CNCCs of the first and second pharyngeal arches at E9.0 (Fakhouri et al., 2017) and Irf6 is expressed in cells of the developing tongue (Goudy et al., 2013). Further, we previously reported that zebrafish expressing the irf6 ${ }^{R 84 C}$ variant under a sox10 promoter exhibit a partial cleft of the anterior neurocranium (Dougherty 
et al., 2013). Together, these results suggest a role of Irf6 in craniofacial development beyond its role in epithelial cell differentiation and fusion.

\section{Disruption of irf6 during neural crest cell migration results in cleft palate in zebrafish}

Germline mutation of irf6 results in early embryonic lethality due to periderm rupture during gastrulation, which precluded evaluation of palate morphogenesis that occurs later in development (Li et al., 2017; Sabel et al., 2009). To circumvent embryonic lethality, we employed an optogenetic gene activation system based on the light-sensitive protein EL222, that serves to induce the expression of genes downstream of the C120 promoter (Motta-Mena et al., 2014). To this end, a dominant-negative form of irf6 consisting of a fusion protein of the irf6 protein-binding domain and the engrailed repressor domain (irf6-ENR) was cloned downstream of the C120 promoter (C120-irf6-ENR; Fig. 4A) (Sabel et al., 2009). When coinjected with VP-16 mRNA, this light activated irf6-ENR construct enabled us to control the timing of irf6 disruption by exposing the embryos to a $465 \mathrm{~nm}$ light-source later in embryogenesis, thereby circumventing gastrulation lethality in the irf6 mutants (Fig. 4A). Zebrafish embryos that were injected with the optogenetic system and continuously exposed to blue light from 10-96 hpf were able to survive, but developed with a slightly curved body axis and a dysmorphic ventral cartilage phenotype, which were not observed in control injected embyros or injected embryos that were raised in the dark (Fig. 4B). Further analysis of the cartilage in these embryos through Alcian blue staining and micro-dissections revealed a cleft in the ANC where a population of cells in the median portion of the ANC was absent (Fig.4C).

\section{Compound homozygote of esrp1 and esrp2 exhibits cleft lip and palate in zebrafish}


To investigate the genetic requirement of esrp1 and esrp2 on zebrafish craniofacial development, CRISPR/Cas9 genome editing was utilized to generate esrp1 and esrp2 mutant alleles. Several alleles of esrp1 and esrp2 were were obtained, where alleles harboring -4bp and $-14 \mathrm{bp}$ indels that lead to frameshift mutations and early protein truncation were selected for breeding, hereafter referred to as esrp $1^{-4 \mathrm{bp} / 4 \mathrm{bp}}$ and esrp2 ${ }^{-14 \mathrm{bp} / 14 \mathrm{bp}}$, respectively (Fig.S4). No phenotype was observed in the esrp $1^{-4 \mathrm{bp} /-4 \mathrm{bp}}$ embryos, and esrp $2^{14 \mathrm{bp} / 14 \mathrm{bp}}$ fish developed normally except that females were infertile, as previously published in independently derived CRISPR alleles of esrp1/2 (Burguera et al., 2017). However, compound homozygote esrp $1^{-4 \mathrm{bp} /-}$ ${ }^{4 \mathrm{bp}} ;$ esrp2 $^{-14 \mathrm{bp} / 14 \mathrm{bp}}$ zebrafish exhibit several phenotypes, consistent with previously published mutants (Burguera et al., 2017). The espr1-4bp/-4bp; esrp2 $^{-14 \mathrm{bp} / 14 \mathrm{bp}}$ embryos also failed to inflate the swim bladder, and the pectoral fins were formed but diminutive and the margins of the fin appeared dysplastic with irregular morphology. Further, Alcian blue staining of double knockouts revealed cleft in the anterior neurocranium, but the ventral cartilages, including the Meckel's cartilage, were formed and appeared wild type (Fig. 5A).

Inter-cross of esrp $1^{-4 \mathrm{bp} /-4 \mathrm{bp}}$; esrp $2^{\mathrm{wt}-14 \mathrm{bp}}$ produces predicted Mendelian ratio of $25 \%$ esrp $1^{-4 \mathrm{bp} /-}$ 4bp; esrp2 ${ }^{-14 \mathrm{bp} / 14 \mathrm{bp}}$ embryos for downstream phenotypic analysis, where $75 \%$ of the embryos appeared wild type. In order to increase the percentage of embryos that can be useful for analysis to $100 \%$, we asked whether morpholino disruption of esrp2 in the esrp $1^{-4 \mathrm{bp} /-4 \mathrm{bp}}$ background would yield consistent a cleft ANC phenotype that phenocopied esrp $1^{-4 \mathrm{bp} /-4 \mathrm{bp}}$; esrp2 $2^{-14 \mathrm{bp} / 14 \mathrm{bp}}$ mutant. In fact, we were successful in phenocopying the cleft ANC phenotype by co-injecting esrp1 and esrp2 MOs into wild type embryos. However, the morpholino concentration needed were relatively high, requiring 2-8 $\mathrm{ng}$ of each $\mathrm{MO}$ to be injected for $\sim 25-$ 
$50 \%$ of embryos to develop a cleft (Fig. 5A,B). However, when esrp $1^{-4 \mathrm{bp} /-4 \mathrm{bp}}$ embryos were injected with esrp2 MO, the cleft ANC phenotype was consistent and observed in nearly $100 \%$ of the embryos, even when the MO concentration was reduced as low as $0.4 \mathrm{ng}$ (Fig. 5A,B). One explanation for this phenomenon is the possibility that transcriptional compensation between esrp1 and esrp2 occurs when each gene is targeted, thereby requiring higher doses of each MO to ablate esrp activity sufficiently (Rossi, 2015 \#1039). But when one of the esrp genes is already disrupted in the homozygous esrp $1^{-4 \mathrm{bp} / 4 \mathrm{bp}}$ mutant, the threshold for full esrp loss of function is lower and requiresld a much smaller dose of $\mathrm{MO}$ to generate the cleft ANC phenotype.

Using scanning electron microscopy (SEM), one can observe that a cleft of the upper margin of the stomodeum invaginates and extends into the cleft ANC. Additionally the keratinocyte morphology of the surface epithelium appeared irregular and round with epithelial blebs in the esrp $^{-4 \mathrm{bp} /-4 \mathrm{bp}} ;$ esrp $^{-14 \mathrm{bp} / 14 \mathrm{bp}}$ embryo. In contrast, the wild type surface epithelium keratinocytes appeared octogonal or hexagonal without epithelial belbs (Fig. 5C). Alizarin red staining of the larvae at $9 \mathrm{dpf}$ also revealed a lack of mineralization at the midline of the parasphenoid bone (Fig. 5D), consistent with a cleft ANC that remained clefted to ossification stage and subsequent larval fish development.

\section{Zebrafish ANC morphogenesis is dependent on epithelial interactions with infiltrating cranial neural crest cells.}

Formation of the zebrafish ANC involves migration of anteromost CNCCs to populate the median portion (frontonasal derived) while more posterior CNCCs migrate from each side 
(maxillary derived), these 3 discrete elements fuse to form the ANC. Concurrent with these cellular movements, the CNCCs undergo differentiation to chondrocytes (Dougherty et al., 2013; Wada et al., 2005). We found that the ablation of irf6 (a key periderm/epithelial gene) and esrp 1/2 (epithelial-restricted genes) both resulted in a cleft in the ANC, where condrocytes are absent along the fusion plane between the frontonasal-derived median element and one side of the maxillary-derived lateral element (Figs. $4 \mathrm{C}$ and $5 \mathrm{~A}$ ).

To investigate the absence of these ANC chondrocytes, we performed lineage tracing of CNCCs in esrp1/2 ablated embryos. Previously, we and others identified that the anteromost cranial neural crest populations at 20 somites that will migrates to and populates the median (frontonasal) element of the ANC (Dougherty et al., 2013; Wada et al., 2005). Accordingly, we labeled the CNCCs at 20-somite stage destined for the median element through photoconversion of kaede under the lineage specificity of the sox 10 promoter. CNCCs of wild type or esrp1/2 CRISPR mutants or esrp1/2 morphants were photo-converted at 12-15 hpf (Fig. $6 \mathrm{~A}, \mathrm{~B})$. Embryos were imaged at $4 \mathrm{dpf}$ to determine the population of the ANC contributed by photo-converted cells. We found that esrp1/2 ablation did not affect the ability of CNCCs to migrate into the ANC, and reached posterior positions, without clustering anteriorly (Fig. 6A,B). Therefore, the cleft of the ANC in the esrp1/2 mutants is not due to an absence of progenitor cells or defect in CNCC migration into the ANC. Nevertheless, Alcian blue staining confirmed that chondrocytes were absent from a cleft in the ANC in the esrp1/2 mutants (Fig. 5A).

To investigate the cellular composition of the ANC cleft, we performed RNAscope ISH staining of coronal cryosections of wild type and esrp1/2 mutants at $4 \mathrm{dpf}$. Sections through ANC 
clefts showed a dense population of cells in the location of the ANC cleft (Fig. 7A,B). In fact, this mass of cells can be localized in the SEM image of the esrp1/2 mutant larvae (Fig. 5C).

These cells are col2a1 negative, consistent with absent Alcian blue staining. Instead, this abberant cell population in the position of the ANC cleft does express irf6 and krt4 (Fig. 7A,B). Confocal images of $5 \mathrm{dpf}$ embryos confirms the expression of irf6 and revealed sox 10 expression in these abberant cells (Fig. 8A). The expression of sox 10 suggests that at least a portion of these cells are CNC-derived whereas krt4 expression indicates epithelial differentiation. The presence of irf6 expression may be indicative of epithelial/periderm cells, or indicative of expression by CNCCs as has been previously reported (Dougherty et al., 2013; Kousa et al., 2019). Based on these results, we hypothesize that epithelial (or possibly periderm) cells that underly the frontonasal and maxillary prominence derivatives are defective in the esrp1/esrp2 null mutants and prevented fusion of the median and lateral elements of the ANC, causing a cleft to form (Fig. 8B). In this way, this is the first direct evidence of cleft pathogenesis in the zebrafish due to epithelial defect, and forms a model to consider conservation of cleft pathogenesis involving the primary palate across vertebrates.

\section{Histological analysis and comparison of $I r f 6^{R 84 C}$ and Esrp1/2 knockout mice.}

Irf6 and Esrp1/2 are established epithelial-expressed genes, and their essential roles in the fusion of craniofacial processes have been reported (Bebee et al., 2015; Ingraham et al., 2006; Iwata et al., 2013). Interestingly, the phenotype of the Irf6-/- and Irf6 ${ }^{R 84 C}$ mouse versus the Esrp $1 / 2^{/-}$mouse differ significantly. Whereas Esrp $1 / 2^{-/}$mice have a clear bilateral cleft lip and primary palate, $I r f 6^{-/}$mice do not exhibit a cleft in the lip. Previous analysis of the $I r f 6^{/-}$and Irf6 ${ }^{R 84 C}$ mouse mutants reported a cleft of the secondary palate and a hypoplastic midface, 
which was attributed to impaired epithelial development (Ingraham et al., 2006; Richardson et al., 2006). However, as we examined the $I r f 6^{R 84 C}$ mutant embryos by histological staining of coronal sections, we found that the lip and premaxilla of the mutant embryo were hypoplastic beyond epithelial associated tissue, where the lip and facial muscles were significantly atrophic, the alveolar bone was deficient, and the incisor roots were displaced (Fig. 9). In contrast, histological analysis of the Esrp1/2 double knockout shows clear development of epithelial and mesenchymal derived tissue with a bilateral lack of fusion between the medial and lateral prominences of the midface (Fig. 9). Therefore, while the $\operatorname{Irf} 6^{R 84 C}$ mutant did not exhibit a cleft of the lip or the primary palate, the midfacial developmental phenotype is more severe.

\section{Genetic interaction of Irf6R84C with Esrp1 and Esrp2}

Irf ${ }^{R 84 C}$ heterozygous mice have some epithelial adhesions; however, these adhesions are reported to resolve and heterozygous mice develop normally (Ingraham et al., 2006;

Richardson et al., 2006). Esrp1 heterozygotes also develop normally, and Esrp2 hetero and homozygous mice have no phenotype. However, the compound ablation of Esrp1 with Esrp2 worsens the cleft lip phenotype observed is the Esrp1 mutant (Bebee et al., 2015).

To test the hypothesis that Irf6 and Esrp1/2 function in the same developmental pathway, we carried out genetic epistasis analysis and generated Irf6;Esrp1;Esrp2 compound mutants. We hypothesized that if Irf6 and Esrp1/2 genetically interacted, that Irf6 and Esrp1 heterozygosity on an Esrp2 null background may result in a cleft phenotype, when Irf6 and Esrp1 heterozygotes do not normally form a cleft. As expected, we observed that $I \mathrm{If} 6^{R 84 C /+} ;$ Esrp $^{1^{+-}}$ 
$;$ Esrp2 $^{+-}$mice developed and reproduced normally. To generate $I r f 6^{R 84 C /+} ;$ Esrp1 $^{+/-} ;$Esrp2 $^{/-}$ embryos, we intercrossed the triple heterozygous mice. We collected a total of 49 embryos from 7 litters from E12.5-E18.5 and tabulated the resulting genotypes (Table 1; Table S1). Based on Mendelian genetics, we expected approximately 3 Irf6 ${ }^{R 84 C /+} ; E s r p 1^{+/-} ; E s r p 2^{/-}$ embryos; however, no triple homozygous mutant embryos were collected (Table 1) suggesting that the genotype is prenatally lethal. Additionally, we did not collect any $\operatorname{Irf6}{ }^{R 84 C / R 84 C} ;$ Esrp $^{-1-}$ embryos, which indicates this genotype is also lethal (Table 1). These findings suggest cummulative lethality between Irf6 and Esrp1/2, where non-lethal genotypes produce a lethal phenotype when combined in the compound mutant.

\begin{tabular}{|c|c|c|c|c|c|}
\hline Irf6 & Esrp1 & Esrp2 & probability & Expected & Observed \\
\hline Het & WT & WT & $1 / 32$ & 1.5 & 3 \\
\hline Het & WT & Het & $1 / 16$ & 3.1 & 4 \\
\hline Het & WT & $\mathrm{KO}$ & $1 / 32$ & 1.5 & 1 \\
\hline Het & Het & WT & $1 / 16$ & 3.1 & 7 \\
\hline Het & Het & Het & $1 / 8$ & 6.1 & 3 \\
\hline Het & Het & KO & $1 / 16$ & 3.1 & 0 \\
\hline Het & $\mathrm{KO}$ & WT & $1 / 32$ & 1.5 & 1 \\
\hline Het & $\mathrm{KO}$ & Het & $1 / 16$ & 3.1 & 5 \\
\hline Het & $\mathrm{KO}$ & $\mathrm{KO}$ & $1 / 32$ & 1.5 & 0 \\
\hline R84C & WT & WT & $1 / 64$ & 0.8 & 0 \\
\hline $\mathrm{R} 84 \mathrm{C}$ & WT & Het & $1 / 32$ & 1.5 & 2 \\
\hline R84C & WT & $\mathrm{KO}$ & $1 / 64$ & 0.8 & 0 \\
\hline $\mathrm{R} 84 \mathrm{C}$ & Het & WT & $1 / 32$ & 1.5 & 3 \\
\hline $\mathrm{R} 84 \mathrm{C}$ & Het & Het & $1 / 16$ & 3.1 & 3 \\
\hline $\mathrm{R} 84 \mathrm{C}$ & Het & KO & $1 / 32$ & 1.5 & 3 \\
\hline R84C & $\mathrm{KO}$ & WT & $1 / 64$ & 0.8 & 0 \\
\hline $\mathrm{R} 84 \mathrm{C}$ & $\mathrm{KO}$ & Het & $1 / 32$ & 1.5 & 0 \\
\hline R84C & $\mathrm{KO}$ & $\mathrm{KO}$ & $1 / 64$ & 0.8 & 0 \\
\hline
\end{tabular}

Irf6R84C/WT; Esrp1+/-; Esrp2+/- triple heterozygous mice were in-crossed and embryos were collected between E12.5 and E21. Table 1 is a subset of expected number of embryos based on Mendelian genetics versus the observed number of viable embryos. The Irf6R84C/WT; Esrp1+/-; Esrp2-/- genotype appears to be lethal prior to E12.5 as approximately 3 embryos were expected but zero embryos were observed. A total of 49 embryos were collected from 7 different litters. 


\section{DISCUSSION}

Orofacial clefts are a common birth defect, and GWASs have identified some critical genes associated with syndromic and non-syndromic cleft. Here we described mouse and zebrafish models using genes with known genetic variants in human cleft patients, IRF6 and ESRP1/2. We present evidence to support that Irf6 and Esrp function in the same regulatory pathway. We observed that mz-irf6 $6^{8 \mathrm{bp} /-8 \mathrm{bp}}$ zebrafish embryos have significantly decreased expression of esrp1 and this is rescued upon introduction of irf6 mRNA. This finding is consistant with esrp1 being a transcriptional target of irf6 and putative irf6 response elements (Khan et al., 2018) can be found surrounding the esrp1 transcriptional start site. Additionally, a RNA-seq experiment identified known irf6 targets including grh/3 and tfap2a. Direct molecular experiments are needed however to test transcriptional regulation of esrp 1 by irf6. We found that Irf6 and Esrp1/2 are consistently co-expressed in the embryonic frontonasal ectoderm and oral epithelium associated with the palate, and epithelium of the mouth opening, in both mouse and zebrafish.

In zebrafish, irf6 null embryos ruptured during gastrulation whereas esrp1/2 null embryos survive to larval stage. However, post-gastrulation ablation of irf6 resulted in a similar cleft morphology of the ANC as the esrp1/2 null. Further analysis of the esrp1/2 null showed that the cleft of the ANC correlated with a cleft in the upper margin of the mouth opening, reminiscent of a human cleft lip. Further, using an NCC-specific photo-convertible reporter line, we were able to show that migration of $\mathrm{CNCC}$ to the developing ANC occured but chondrogenesis was impaired. 
This work highlights the utility of complementary studies of palate morphogenesis in zebrafish and mouse models. The zebrafish model affords the transgenic tractability and visualization of CNCC migration, enabling us to determine the cellular mechanism responsible for the cleft ANC. The mouse mutants provide the mammalian anatomic contexts to examine cleft malformation. Irf6 ${ }^{R 84 C}$ mice have significant midfacial hypoplasia and oral occlusion when compared to Esrp1/2 null embryos that exhibit bilateral cleft lip and palate. Histological examination of $\operatorname{Irf} 6^{R 84 C}$ mice revealed loss of portions of normal mesenchymal-derived tissue (muscle, nerve, bone, and vascularity). In contrast, the mesenchymal derived tissues are fully formed but displaced in the Esrp1/2 null mice.

The early lethality of irf6 null zebrafish at the onset of gastrulation initially precluded analysis of the irf6 zygotic requirement in craniofacial development. Here, we utilized an optogenetics strategy to disrupt irf6 function after gastrulation when the embryonic body axis had formed, thereby revealing the zygotic requirement for irf6. Future studies will use the irf6 optogenetic model to study the roles of irf6 during ANC and lip morphogenesis. Interestingly, periderm markers identified in the mouse lambdoidal junction were found to be dysregulated in the irf6 mutant zebrafish model, specifically grh/3, tfap2a and perp. Additionally gata3, which was identified as a mesenchymal marker at the fusion zone of mice (Li et al., 2019), is dysregulated in the irf6 null zebrafish.

While the esrp $1^{-4 b p /-4 b p} ;$ esrp2 ${ }^{-14 b p /-14 b p}$ zebrafish exhibited a consistent cleft lip and palate phenotype, the infertility of the esrp $2^{-14 b p /-14 b p}$ fish preclude large-scale experiments to analyze downstream mechanisms of the development of cleft palate. We generated a robust esrp $1^{-4 \mathrm{bp} /-}$ 
4bp; esrp2 morphant assay that can be applied to toward chemical screening experiments and functional testing of human ESRP1/2 gene variants.

In humans, CPO is less common than CL/P (Bush and Jiang, 2012; Gritli-Linde, 2008; Van Otterloo et al., 2016). Although humans and mice share approximately $99 \%$ of their genes and the early craniofacial development of the mouse embryo closely mirrors the human, including gene expression (Swartz et al., 2011), there is a striking difference in the manifestation of orofacial cleft defects (Gritli-Linde, 2008). Most often when a human CL/P-associated gene has been disrupted in mice, a cleft of the palate forms but the lip appears normal. Our current understanding in humans is that CL/P and CPO are different genetic disorders (Dixon et al., 2011; Gritli-Linde, 2008; Juriloff and Harris, 2008). These discrepencies between humans and mouse models hampens understanding of the etiopathogenesis of human CL/P. Here we characterize the Esrp1/2 null mouse, exhibiting bilateral CL/P, as an important model for studying OFC etiopathogenesis. Additionally, as we place ESRP1 in the IRF6 gene regulatory pathway, we hope to better understand how alternative isoforms regulated by ESRP1 may in turn be important for palate development.

Whereas zebrafish have historically been an excellent model organism for forward genetic screens, CRISPR/Cas9 gene editing technology has permitted relatively efficient reverse genetic engineering of zebrafish (Liu et al., 2019). This utility of the zebrafish embryo for studying developmental processes and modeling human cleft-associated genes necessitates further study into their craniofacial morphogenesis. Transplant and lineage tracing experiments have illuminated the neural crest origin of the zebrafish ANC, and how the 
frontonasal and paired maxillary cartilage elements converge into a continuous cartilage structure (Dougherty et al., 2013; Dougherty et al., 2012; Wada et al., 2005). We show that IRF6 and ESRP1 are conserved in their requirement for ANC morphogenesis, where disruption result in orofacial cleft in human, mouse and zebrafish. These findings provide evidence of conserved molecular and morphological processes occurring in the merging and fusion of the mouse and zebrafish midface.

We suspect that non-epithelial expression of Irf6 contributes to normal craniofacial morphogenesis and may explain some differences in the Irf6 and Esrp mutant phenotypes. Future research utilizing tissue-specific knockout of Irf6 will address this hypothesis. We also suspect that the Irf6 phenotype is more severe because Irf6 acts upstream of Esrp1, along with additional targets, and ongoing experiments on the transcriptional activity of Irf6 will be important. Continued breeding of the triple heterozygous $I r f 6^{R 884 C /+}, \operatorname{Esrp}^{1^{+-}} ; \mathrm{Esrp}^{\mathrm{T}^{+/}}$mice will also test a genetic epistasis interaction between them. Recently an in-depth analysis of a lineage-specific Esrp1 knockout mouse was completed and found that Esrp1 regulates proliferation of the mesenchyme of the lateral nasal prominences, along with being required for fusion of the medial and lateral nasal prominences (Lee et al., 2020). The Esrp1/2 knockout mouse is an exciting model for studying craniofacial development and the etiology of CL/P, as relatively few mouse models exhibit cleft lip phenotype. Ongoing work to identify Esrp1/2 molecular targets and mechanistic studies of these targets will provide new insight into palate morphogenesis. 


\section{METHODS}

\section{Animal breeding and gene editing}

All animal experiments were performed in accordance with protocols approved by

Massachusetts General Hospital Animal Care and Usage Committee. C57BI/6J (wild type) animals were obtained from the Jackson Laboratory. $I r f 6^{R 84 C /+}$ mice were received as a gift from Dr. Yang Chai. Esrp1 ${ }^{+/} ;$Esrp2 $^{-/}$mice were received from Dr. Russ Carstens. Embryonic day 0.5 was considered to be noon on the day of the copulatory plug.

Zebrafish (Danio rerio) adults and embryos were maintained in accordance with approved institutional protocols at Massachusetts General Hospital. Embryos were raised at $28.5^{\circ} \mathrm{C}$ in E3 medium (5.0 mM NaCl, $\left.0.17 \mathrm{mM} \mathrm{KCl}, 0.33 \mathrm{mM} \mathrm{CaCl}_{2}, 0.33 \mathrm{mM} \mathrm{MgSO}_{4}\right)$ with $0.0001 \%$ methylene blue. Embryos were staged according to standardized developmental timepoints by hours or days post fertilization (hpf or dpf, respectively).(Kimmel et al., 1995) All zebrafish lines used for experimentation were generated from the Tübingen strain.

CRISPR sgRNA target sites were identified by a variety of online CRISPR computational programs such as zifit.partners.org/ZiFiT(Sander et al., 2007), crispr.mit.edu (Ran et al., 2013), and chopchop.rc.fas.harvard.edu.(Montague et al., 2014) sgRNAs were designed with the traditional sequence constraint of a 3' PAM sequence containing NGG and an additional sequence constraint of a 5' NG for in vitro RNA synthesis.

The esrp1, esrp2 and irf6 CRISPR sgRNAs were generated by in vitro transcription from a SP6 promoter as described.(Gagnon et al., 2014) Lyophilized Cas9 protein (PNA Bio) was resuspended in $\mathrm{ddH}_{2} \mathrm{O}$ to a stock concentration of $1 \mu \mathrm{g} / \mu \mathrm{l}$ and stored in single-use aliquots in $80^{\circ} \mathrm{C}$ to avoid freeze-thaw inactivation and kept for 6 months. One-cell staged zebrafish embryos were microinjected directly in the cytoplasm with $2 \mathrm{nl}$ of a solution containing $15 \mathrm{ng} / \mu \mathrm{l}$ 
of sgRNA and $100 \mathrm{ng} / \mu \mathrm{l}$ of Cas9 protein pre-complexed for 5-10 minutes at room temperature. A subset of embryos injected with the sgRNA and Cas9 protein mixture was harvested for genomic DNA to confirm the presence of indels, and the rest were grown into adulthood as F0 mosaic fish. F0 adult fish were subsequently outcrossed with wild-type fish to generate F1 founders with germline transmission of indel alleles. F1 founders were further outcrossed with wild-type fish to yield a large number of heterozygote fish, and minimize the presence of offtarget edits. Lastly, F2 heterozygotes were incrossed to generate homozygote embryos for phenotypic analysis.

Genomic DNA for genotyping was isolated from either whole $24 \mathrm{hpf}$ embryos or tail fin clips using the HotSHOT method as described.(Meeker et al., 2007) Genotyping primers flanking the CRISPR sgRNA site were designed using a combination of ChopChop (chopchop.rc.fas.harvard.edu) and NCBI primer BLAST (ncbi.nlm.nih.gov/tools/primer-blast/). Forward primers were synthesized by Invitrogen with 5'-FAM modifications. Microsatellite sequencing analyses were used to determine indel mutation sizes and frequencies (MGH DNA Core), and Sanger sequencing was performed on PCR amplicons of CRISPR sgRNA to confirm the exact sequence changes resulting from CRISPR mutagenesis.

\section{mRNA sequencing and qPCR}

Total RNA was isolated from $4 \mathrm{hpf}$ wild type and maternal-null irf6 ${ }^{-/}$embryos by TRIzol and phenol-chloroform ethanol precipitation, as previously described. Total RNA was quantified with the Nanodrop 2500 and assessed for quality with Bioanalyzer 2100 RNA chips (Agilent). Samples with RNA integrity numbers (RIN) over 9 were selected to proceed with sequencing library preparation. mRNA-seq libraries were prepared with the NEBNext Ultra RNA library 
preparation kit with poly $(A)$ mRNA magnetic isolation module (NEB) essentially according to manufacturer protocols. Resulting cDNA libraries were quantified by a Qubit fluorometer and assessed for quality with a Bioanalyzer. The sequencing-ready cDNA libraries were quantified with the NEBNext library quantification kit for Illumina (NEB). mRNA-seq libraries were sequenced with single-end 50 at $\approx 20$ million reads per sample with biological triplicates. For qPCR, 30 embryos per sample were collected in RLT buffer (Qiagen) and flash-frozen in liquid nitrogen. Samples were homogenized using a rotor-stator homogenizer, and RNA was isolated using an RNeasy Mini Kit (Qiagen). Total mRNA was quantified using a Nanodrop spectrophotometer and used for cDNA synthesis (Thermo). qPCR was performed with Taqman probes and reagents (Thermo), and expression was normalized to 18s rRNA expression.

\section{Zebrafish embryo microinjection of mRNA and morpholinos}

Microinjection of mRNA was performed by injecting $2 \mathrm{nl}$ of $\mathrm{mRNA}$ solution with $0.05 \%$ phenol red directly into the cytoplasm of one-cell staged embryos. Lyophilized morpholinos were resuspended with ddH2O to a stock concentration of $20 \mathrm{ng} / \mu \mathrm{l}$ and stored at $\mathrm{RT}$ in aliquots. Individual aliquots were heated to $70^{\circ} \mathrm{C}$ and briefly vortexed before preparation of the injection mix to ensure full dissolution. Mismatch control morpholinos were injected under identical conditions to control for potential toxicities. Embryos from all methods of microinjection were examined at $3 \mathrm{hpf}$ to remove unfertilized embryos, which were quantified against the total number of microinjected embryos to ensure no fertilization defects were observed.

\section{Whole-mount in situ hybridization}


Embryos were isolated at various time points and fixed in $4 \%$ formaldehyde at $4^{\circ} \mathrm{C}$ for $12-16$ hrs. Subsequently, embryos were washed and stored in methanol. WISH and DIG-labeled riboprobes were synthesized as described.(Thisse and Thisse, 2008) Briefly, for riboprobe synthesis, PCR was performed using embryonic cDNA as templates and T7 promoter sequence-linked reverse primers to generate cDNA templates for in vitro transcription. PCR reactions were purified using the NucleoSpin gel and PCR clean-up kit (Machery-Nagel). In vitro transcription was performed using a T7 polymerase (Roche) and DIG labeling mix (Roche). DIG-labeled riboprobes were isolated with ethanol-NaOAc precipitation, resuspended in DEPC-treated $\mathrm{dd}_{2} \mathrm{O}$, and stored at $-20^{\circ} \mathrm{C}$. All PCR products were TOPO cloned into pGEM-T Easy vectors (Promega) and sequence verified by Sanger sequencing. WISH colorimetric signal detection was performed using an alkaline phosphatase-conjugated antiDIG antibody (Roche) and BM Purple AP substrate (Roche).

\section{RNAscope in situ hybridization}

Zebrafish and mouse embryos were fixed in $4 \%$ formaldehyde, taken through a sucrose gradient and cryo-embedded and sectioned. Probes were designed and purchased from ACD Bio, and hybridization and staining was performed according to the manufacturer's protocol. Stained sections were imaged using either a confocal microscope, where a z-stack was obtained and analyzed on ImageJ for z-stack maximum intensity projections or a standard fluorescent microscope.

\section{Skeletal staining and brightfield imaging}


Zebrafish embryos were fixed at $96 \mathrm{hpf}$ or $120 \mathrm{hpf}$ in $4 \%$ formaldehyde and stored at $4^{\circ} \mathrm{C}$ overnight, washed with PBS, dehydrated in 50\% ethanol, and stained with acid-free Alcian blue overnight on a rotating platform at RT as described.(Thisse and Thisse, 2008) Stained embryos were washed with ddH2O and subsequently bleached $(0.8 \% \mathrm{~W} / \mathrm{V} \mathrm{KOH}, 0.1 \%$ Tween $20,0.9 \% \mathrm{H} 2 \mathrm{O} 2$ ) until cell pigmentation was no longer present. For double-stained embryos with Alcian blue and alizarin red, embryos were stained with a $0.05 \%$ alizarin red solution in ddH2O for 30 minutes on a rotating platform at $\mathrm{RT}$ following bleaching with $\mathrm{KOH}$ and $\mathrm{H} 2 \mathrm{O} 2$. Afterward, double-stained embryos were placed in three changes of a tissue-clearing solution consisting of $25 \%$ glycerol and $0.1 \% \mathrm{KOH}$, each for $25 \mathrm{~min}$. Whole and dissected stained embryos were mounted in 3\% methylcellulose on a depression slide and imaged using a Nikon Eclipse 80i compound microscope with a Nikon DS Ri1 camera. Z-stacked images were taken to increase the depth-of-field with the NIS Element BR 3.2 software. Stacked images were processed by ImageJ to generate maximum intensity projection images.

For scanning electron microscopy (SEM), 4 dpf embryos were fixed in half-strength Karnovsky fixative. Samples were processed, and images were obtained by CBSET, Inc. Lexington, MA.

\section{Optogenetic expression of irf6 in zebrafish}

Genes irf6, irf6-ENR, irf6 ${ }^{R 84 C}$, and mCherry were isolated by PCR from various templates and inserted into the pGL4.23-(C120x5)-TATA vector with In-Fusion cloning (Clontech) according to manufacturer instructions using a 1:2 vector-to-insert ratio to generate optogenetic response plasmids. The constructs were transformed in Stellar chemically competent cells (Clontech), and colonies were screened by PCR, restriction digests, Sanger sequencing, and whole plasmid sequencing to verify the sequence identities and accuracy of the constructs. Light- 
sensitive response protein VP16-EL222 was subcloned into pCS2+8 and in vitro transcribed from the SP6 promoter as described above to generate capped mRNA for embryo microinjections. The optogenetics injection mix was comprised of $25 \mathrm{ng} / \mu \mathrm{l} \mathrm{EL222}$ and $10 \mathrm{ng} / \mu \mathrm{l}$ pGL4.23 response plasmid with $0.05 \%$ phenol red. Each embryo was microinjected with $2 \mathrm{nl}$ of the optogenetics injection mix directly in the cytoplasm at the one-cell stage, immediately wrapped in aluminum foil, and placed into a dark incubator. Unfertilized and abnormal embryos were removed at $3 \mathrm{hpf}$ in the dark room with limited exposure to ambient light. Injected embryos were divided into two groups (dark and light) at the desired developmental stage in E3 media without methylene blue and placed under 465nm blue light (LED panel, HQRP) at $0.3 \mathrm{~mW} / \mathrm{cm} 2$ (measured by a PM100D digital power meter with an SV120VC photodiode power sensor, ThorLabs) with constant illumination. Control embryos containers were wrapped in aluminum foil.

\section{Lineage Tracing}

Embryos originating from an espr1-4bp/-4bp $;$ esrp2 $2^{+/ 14 \mathrm{bp}}$ incross, wild type embryos injected with 8ng esrp1 MO and 4ng esrp2 MO at the one-cell stage, or uninjected wild-type embryos, all in a $\operatorname{Tg}($ sox 10:kaede) background were grown until 20 somites, oriented for imaging in the sagittal position, and encased in 1\% low-melt agarose. Using the 405nm UV laser and ROI setting in a Leica SP8 confocal microscope, the anterior-most portion of NCCs that contribute to the FNP were unilaterally photoconverted, keeping the alternate side as an internal control, as previously described.(Dougherty et al., 2012) Photoconverted embryos were carefully microdissected out of the agar, and grown in E3 at $28.5^{\circ} \mathrm{C}$ until $4 \mathrm{dpf}$ and imaged again to track the photoconverted cells. Maximum-projections of the photoconverted half of the embryo, or 
the planes consisting of the palate, in $14 \mathrm{hpf}$ or $4 \mathrm{dpf}$ embryos, respectively, were generated using Fiji/lmageJ.

\section{Acknowledgement}

We ae grateful to Jessica Bethoney for excellent management of our aquatics facility. We thank Irimia Manuel for willingness to share an esrp1/2 zebrafish mutant alleles (did not survive shipment, not used in this study) and Yang Chai for sharing the Irf6 ${ }^{R 84 C}$ mouse mutant allele. The Esrp1 and Esrp2 mouse alleles were generated by R.C. with funding from the National Institutes of Health (R01DE024749). This work was supported by grants to E.C.L. from the National Institutes of Health (R01DE027983), the Shriners Hospital for Children, and the Laurie and Mason Tenaglia Massachusetts General Research Scholar Award. 


\section{Figure Legends}

\section{Figure 1. esrp1 expression is downregulated in irf6 null zebrafish embryos. (A)}

Hierarchical clustering of top differentially expressed genes (DEGs) defined by RNAsequencing performed on WT vs. mz-irf6 ${ }^{-8 b p /-8 b p}$ (irf6-/-) zebrafish embryos at 4-5hpf. Top DEGs were identified by selecting genes with an adjusted p-value (Benjamini-Hochberg) $<0.01$ and absolute log2-fold change $>2$. Data shown for 3 biological replicates. Color scale at top left represents relative levels of expression with yellow showing higher expression levels and blue showing lower expression. (B) Volcano plot from the RNA-seq dataset showing the distribution of DEGs based on p-values and log2-fold change. Previously published irf6regulated genes are expressed at significantly higher level in wild type relative to mz-irf6-/-, including $g r h / 3, k l f 17$, and wnt11. The newly identified cleft-associated gene esrp1 is also expressed significantly higher in wild type relative to irf6-/-. Horizontal and vertical lines represent the p-value cutoff of 0.01 and the log2-fold change cutoff of 2 , respectively. (C) Gene ontology (GO) gene-concept network analysis of RNA-seq data showing that irf6-/- embryos have perturbations in processes such as transcription factor activity, signal receptor binding, and structural molecule activity. Note that many of these genes, such as wnt11, fgf8, $\operatorname{tg} f b 1$, krt4, and krt5, are implicated in ectoderm development and cell specification. Grey nodes show GO terms, colored nodes show individual genes from the RNA-seq dataset, and edges connect genes to one or more associated GO terms. Colored nodes show relative enrichment (measured by fold-change) of genes in wild type samples relative to irf6-/- embryos. Maps were generated using the enrichplot package in R. (D) qPCR gene expression analysis for esrp1, showing approximately 6-fold esrp1 downregulation in mz-irf6 ${ }^{8 \mathrm{bp} /-8 \mathrm{bp}}$ embryos compared 
to wild type at $4 \mathrm{hpf}$, and rescued esrp1 gene over-expression in mz-irf6 ${ }^{-8 \mathrm{bp} /-8 \mathrm{bp}}$ embryos injected with wild type zebrafish irf6 mRNA. $n=4 .{ }^{*} \mathrm{p}<0.05$.

Figure 2. irf6, esrp1 and esrp2 are co-expressed in the oral epithelium of zebrafish embryos. (A-C) Whole mount in situ hybridization showing irf6, esrp1 and esrp2 maternal deposited transcripts are detected at the 8-cell and shield stage (A; arrow depicts periderm) circumscribes the developing stomodeum and lines the oral epithelium of zebrafish embryos at 48 (B) and 72 (C) hpf (depicted by arrow). All whole-mount embryos are oriented with anterior toward left of page, dorsal toward top of page. Coronal sections of 48 (D) and 72 (E) hpf embryos analyzed by RNAscope in situ hybridization, (dorsal toward top of page) showing cellular RNA co-expression of irf6 (green) and esrp1 (white) in surface and oral epithelial cells. sox10 (red) staining depicts cartilage elements of the palate. White arrow depicts area of fusion between Meckel's cartilage elements. Scale bars: $250 \mu \mathrm{m}(\mathrm{A})$ and $100 \mu \mathrm{m}(\mathrm{B}-\mathrm{E})$.

\section{Figure 3. Irf6, Esrp1 and Esrp2 are co-expressed in the oral epithelium of mouse}

embryos. (A) Whole mount in situ hybridization of E10.5 embryos showing Irf6, Esrp1 and Esrp2 mRNA expression in the surface epithelium and concentrated within the ectoderm of the frontonasal prominences and first brachial arch. Oblique and frontal orientation. Scale bars: $500 \mu \mathrm{m}$. (B-F) Sections of (B) E10, (C,D) E11.5, (E) E13.5 and (F) E15 embryos analyzed by RNAscope in situ hybridization showing mRNA cellular co-expression of Irf6 (green), Esrp1 (red) and Esrp2 (white) in the surface ectoderm (E10), lining the frontonasal and maxillary prominences, including expression in periderm (arrows) (E11.5) and lining the palatal shelves (E13.5, E15). (B) sagittal and (C-F) coronal sections. Scale bars: $100 \mu \mathrm{m}$. 
Figure 4. EL222 optogenetic disruption of irf6 circumvents early embryonic lethality and causes a cleft palate phenotype. (A) Schematic of EL222 Optogenetic system. VP16-EL222 monomers are inactive under dark conditions. Upon stimulation by 465nm light, VP16-EL222 dimerizes, drives gene expression downstream of the C120 promoter, and induces the expression of a dominant negative form of irf6 (irf6-ENR). Embryos were exposed to blue light from 10-72 hpf to circumvent embryonic lethality in mz-irf6 ${ }^{8 \mathrm{bp} /-8 \mathrm{bp}}$ embryos. (B-E) Brightfield microscopy of 72 hpf zebrafish embryos injected with the optogenetic system and grown in the dark (D) or exposed to blue light from 10-72 hpf (E) compared to control injected embryos (B, C). Injected fish exposed to blue light exhibit retrusion of the midface (arrowhead) and curved body not observed in the other groups. (F-Q) Alcian blue staining of cartilage and microdissection of the palate of 72 hpf embryos reveals a midface retrusion and cleft phenotype through the medial ethmoid plate (panel Q arrowhead) in the C120-irf6-ENR injected embryos grown under blue light (O-Q) which is not seen in control injected embryos (IK) or injected embryos grown in the dark (L-N). Scale bars: 150um.

Figure 5. esrp1/2 double mutants display a cleft lip and palate. (A) Alcian blue staining of 4 dpf zebrafish. Representative images of wild type, esrp1 CRISPR mutant (esrp1 -/-), and esrp1/2 double CRISPR mutant (esrp1 -/-; esrp2 -/-), as well as esrp1 CRISPR mutant treated with esrp2 morpholino and wild type treated with esrp1 and esrp2 morpholino (esrp1 MO, esrp2 MO). Flat-mount images of the anterior neurocranium (ANC) show a cleft (arrow) between the median element and lateral element of the ANC when both esrp1 and esrp2 function were disrupted. Lateral images and flat-mount images of the ventral cartilage (VC) 
show only subtle changes in morphology between WT and esrp1/2 -/- zebrafish. (B) Morphant phenotypes observed over a range of esrp1 and esrp2 morpholino doses. Single esrp2 MO injections in the esrp1-/- background achieves nearly 100\% phenotype penetrance, even at very low MO doses. (C) SEM of 5 dpf zebrafish showing discontinuous upper lip (closed arrow) in the esrp1/2 double CRISPR mutant as well as absent preoptic cranial neuromasts (open arrow) and abnormal keratinocyte morphology. White arrow depicts aberrant cell mass

(D) Representative images of alizarin red/Alcian blue staining of $9 \mathrm{dpf}$ esrp1/2 double CRISPR mutant zebrafish and wild type clutch-mate controls. Esrp1/2 ablation causes abnormal morphology of the mineralizing parasphenoid bone, where the bone appears wider and with a cleft (arrow).

Figure 6. esrp1/2-null cranial neural crest cells migrate to the anterior neurocranium but do not differentiate to chondrocytes. (A) Lineage tracing of wild type or esrp1/2 morphant zebrafish embryos using the $\operatorname{Tg}($ sox10:kaede) line, native kaede fluorescence is shown in green, and photo-converted kaede is shown in magenta. Sagittal and horizontal views of zebrafish embryos at $19 \mathrm{hpf}$ and $4.5 \mathrm{dpf}$, respectively. The anterior-most neural crest frontonasal prominence (FNP) progenitors were photoconverted at $19 \mathrm{hpf}$. At $4.5 \mathrm{dpf}$, the wild type signal tracks to the medial portion of the anterior neurocranium (ANC). Both the esrp1/esrp2 double CRISPR mutants and esrp1/2 morphants exhibit a cleft in the ANC with absence of a portion of sox10-positive cells in the medial portion of the ANC, but the labeled CNCC representing FNP progenitors did reach and populate the entire length of the ANC. (B) Illustrative summary of lineage tracing results showing that photo-converted anterior most CNCCs contributing to FNP do migrate into the ANC in esrp1/2 mutant embryos but a cleft 
forms at the juxtaposition of the FNP-derived median element and the maxillary-derived lateral element.

\section{Figure 7. Anterior neurocranium of esrp1/2 double mutants is populated by} undifferentiated cells. Representative images of RNAscope ISH of coronal sections of esrp1/2 double CRISPR mutants and wild type clutch-mate controls at $4 \mathrm{dpf}$. (A) Sections through ANC anterior to the eyes. col2a1 (red) staining depicts normal morphology of the ANC cartilage elements in wild type while a cleft is apparent in the esrp1/2-/- zebrafish, with dapi (blue) stained cells between adjacent trabeculae (white arrow). These col2a1 negative cells do not express epithelial markers krt4 (cyan) or krt5 (magenta), however there is low expression of irf6 (green; white box). (B) Sections posterior to those in (A) show col2a1 negative cells continuing inferior to the trabeculae in the esrp1/2 mutant zebrafish and cells have low expression of irf6 and krt4 (white box). Scale bars: $20 \mu \mathrm{m}$.

\section{Figure 8. Aberrant anterior neurocranium cells of esrp1/2 double mutants express}

CNCC and epithelial cell markers. Representative z-stacks of RNAscope ISH of coronal sections of esrp 1/2 double CRISPR mutants and wild type clutch-mate controls at $5 \mathrm{dpf}$. Sections through ANC anterior to the eyes. ANC cartilage elements are outlined in white. col1a1 (white) staining depicts perichondrium surrounding the abberant mass of cells in the esrp1/2 mutant zebrafish consistant with chondrogenic condensation (arrow). IIf6 (green) and sox10 (red) expression is apparent in these cells (arrow head). Dapi (blue). Scale bar: $20 \mu \mathrm{m}$. (B) Illustrative summary of RNAscope ISH results showing a heterogenous mix of cells populating the cleft between ANC cartilage elements. 
Figure 9. Irf6 ${ }^{R 84 C / R 84 C}$ and Esrp1/2 dKO mice display different cleft characteristics.

Hematoxylin and eosin staining of coronal cryosections of wild type, Irf6 ${ }^{R 84 C / R 84 C}$ and Esrp1/2 dKO. (A-F) E13.5 asterisk: tooth primordium, closed arrowhead: muscle, open arrowhead: vibrissae. (G-I) Magnified images of E13.5 lip tissue showing differences in tissue composition. (J-L) E15.5 sections through secondary palate shelves. Both Irf6 ${ }^{R 84 C / R 84 C}$ and Esrp1/2 dKO mice exhibit a secondary cleft with impaired elevation of the palatal shelves. However, whereas failed elevation in $\operatorname{Irf} 6^{R 84 C / R 84 C}$ mice has been attributed to eplithelial adhesions, Esrp1/2 dKO mice do not exhibit adhesion. Scale bars: $100 \mu \mathrm{m}$. 


\section{REFERENCES}

Abramyan, J., and Richman, J.M. (2015). Recent insights into the morphological diversity in the amniote primary and secondary palates. Developmental dynamics : an official publication of the American Association of Anatomists 244, 1457-1468.

Beaty, T.H., Marazita, M.L., and Leslie, E.J. (2016). Genetic factors influencing risk to orofacial clefts: today's challenges and tomorrow's opportunities. F1000Research 5, 2800. Bebee, T.W., Park, J.W., Sheridan, K.I., Warzecha, C.C., Cieply, B.W., Rohacek, A.M., Xing, Y., and Carstens, R.P. (2015). The splicing regulators Esrp1 and Esrp2 direct an epithelial splicing program essential for mammalian development. eLife 4.

Botti, E., Spallone, G., Moretti, F., Marinari, B., Pinetti, V., Galanti, S., De Meo, P.D., De Nicola, F., Ganci, F., Castrignano, T., et al. (2011). Developmental factor IRF6 exhibits tumor suppressor activity in squamous cell carcinomas. Proceedings of the National Academy of Sciences of the United States of America 108, 13710-13715.

Burguera, D., Marquez, Y., Racioppi, C., Permanyer, J., Torres-Mendez, A., Esposito, R., Albuixech-Crespo, B., Fanlo, L., D'Agostino, Y., Gohr, A., et al. (2017). Evolutionary recruitment of flexible Esrp-dependent splicing programs into diverse embryonic morphogenetic processes. Nature communications $8,1799$.

Bush, J.0., and Jiang, R. (2012). Palatogenesis: morphogenetic and molecular mechanisms of secondary palate development. Development 139, 231-243.

Cordero, D.R., Brugmann, S., Chu, Y., Bajpai, R., Jame, M., and Helms, J.A. (2011). Cranial neural crest cells on the move: their roles in craniofacial development. American journal of medical genetics Part A 155A, 270-279.

Cox, L.L., Cox, T.C., Moreno Uribe, L.M., Zhu, Y., Richter, C.T., Nidey, N., Standley, J.M., Deng, M., Blue, E., Chong, J.X., et al. (2018). Mutations in the Epithelial Cadherin-p120-Catenin Complex Cause Mendelian Non-Syndromic Cleft Lip with or without Cleft Palate. American journal of human genetics 102, 1143-1157.

Creuzet, S., Couly, G., and Le Douarin, N.M. (2005). Patterning the neural crest derivatives during development of the vertebrate head: insights from avian studies. Journal of anatomy 207, 447-459.

de la Garza, G., Schleiffarth, J.R., Dunnwald, M., Mankad, A., Weirather, J.L., Bonde, G., Butcher, S., Mansour, T.A., Kousa, Y.A., Fukazawa, C.F., et al. (2013). Interferon regulatory factor 6 promotes differentiation of the periderm by activating expression of Grainyheadlike 3. The Journal of investigative dermatology 133, 68-77.

Dixon, M.J., Marazita, M.L., Beaty, T.H., and Murray, J.C. (2011). Cleft lip and palate: understanding genetic and environmental influences. Nature reviews Genetics 12, 167-178. Dougherty, M., Kamel, G., Grimaldi, M., Gfrerer, L., Shubinets, V., Ethier, R., Hickey, G., Cornell, R.A., and Liao, E.C. (2013). Distinct requirements for wnt9a and irf6 in extension and integration mechanisms during zebrafish palate morphogenesis. Development 140, 76 81.

Dougherty, M., Kamel, G., Shubinets, V., Hickey, G., Grimaldi, M., and Liao, E.C. (2012). Embryonic fate map of first pharyngeal arch structures in the sox10: kaede zebrafish transgenic model. The Journal of craniofacial surgery 23, 1333-1337.

Duncan, K.M., Mukherjee, K., Cornell, R.A., and Liao, E.C. (2017). Zebrafish models of orofacial clefts. Developmental dynamics : an official publication of the American Association of Anatomists 246, 897-914. 
Fakhouri, W.D., Metwalli, K., Naji, A., Bakhiet, S., Quispe-Salcedo, A., Nitschke, L., Kousa, Y.A., and Schutte, B.C. (2017). Intercellular Genetic Interaction Between Irf6 and Twist1 during Craniofacial Development. Scientific reports 7, 7129.

Ferretti, E., Li, B., Zewdu, R., Wells, V., Hebert, J.M., Karner, C., Anderson, M.J., Williams, T., Dixon, J., Dixon, M.J., et al. (2011). A conserved Pbx-Wnt-p63-Irf6 regulatory module controls face morphogenesis by promoting epithelial apoptosis. Developmental cell 21, 627-641.

Gagnon, J.A., Valen, E., Thyme, S.B., Huang, P., Akhmetova, L., Pauli, A., Montague, T.G., Zimmerman, S., Richter, C., and Schier, A.F. (2014). Efficient mutagenesis by Cas 9 proteinmediated oligonucleotide insertion and large-scale assessment of single-guide RNAs. PloS one 9, e98186.

Goodwin, A.F., Kim, R., Bush, J.O., and Klein, O.D. (2015). From Bench to Bedside and Back: Improving Diagnosis and Treatment of Craniofacial Malformations Utilizing Animal Models. Current topics in developmental biology 115, 459-492.

Goudy, S., Angel, P., Jacobs, B., Hill, C., Mainini, V., Smith, A.L., Kousa, Y.A., Caprioli, R., Prince, L.S., Baldwin, S., et al. (2013). Cell-autonomous and non-cell-autonomous roles for IRF6 during development of the tongue. PloS one 8, e56270.

Gritli-Linde, A. (2008). The etiopathogenesis of cleft lip and cleft palate: usefulness and caveats of mouse models. Current topics in developmental biology 84, 37-138.

Helms, J.A., Cordero, D., and Tapadia, M.D. (2005). New insights into craniofacial morphogenesis. Development 132, 851-861.

Hu, D., Marcucio, R.S., and Helms, J.A. (2003). A zone of frontonasal ectoderm regulates patterning and growth in the face. Development 130, 1749-1758.

Ingraham, C.R., Kinoshita, A., Kondo, S., Yang, B., Sajan, S., Trout, K.J., Malik, M.I., Dunnwald, M., Goudy, S.L., Lovett, M., et al. (2006). Abnormal skin, limb and craniofacial morphogenesis in mice deficient for interferon regulatory factor 6 (Irf6). Nature genetics 38, 1335-1340.

Iwata, J., Suzuki, A., Pelikan, R.C., Ho, T.V., Sanchez-Lara, P.A., Urata, M., Dixon, M.J., and Chai, Y. (2013). Smad4-Irf6 genetic interaction and TGFbeta-mediated IRF6 signaling cascade are crucial for palatal fusion in mice. Development 140, 1220-1230.

Jiang, R., Bush, J.O., and Lidral, A.C. (2006). Development of the upper lip: morphogenetic and molecular mechanisms. Developmental dynamics : an official publication of the American Association of Anatomists 235, 1152-1166.

Juriloff, D.M., and Harris, M.J. (2008). Mouse genetic models of cleft lip with or without cleft palate. Birth defects research Part A, Clinical and molecular teratology 82, 63-77.

Khan, A., Fornes, 0., Stigliani, A., Gheorghe, M., Castro-Mondragon, J.A., van der Lee, R., Bessy, A., Cheneby, J., Kulkarni, S.R., Tan, G., et al. (2018). JASPAR 2018: update of the openaccess database of transcription factor binding profiles and its web framework. Nucleic acids research 46, D1284.

Kimmel, C.B. (1989). Genetics and early development of zebrafish. Trends in genetics : TIG 5, 283-288.

Kimmel, C.B., Ballard, W.W., Kimmel, S.R., Ullmann, B., and Schilling, T.F. (1995). Stages of embryonic development of the zebrafish. Developmental dynamics : an official publication of the American Association of Anatomists 203, 253-310.

Knight, A.S., Schutte, B.C., Jiang, R., and Dixon, M.J. (2006). Developmental expression analysis of the mouse and chick orthologues of IRF6: the gene mutated in Van der Woude 
syndrome. Developmental dynamics : an official publication of the American Association of Anatomists 235, 1441-1447.

Knight, R.D., and Schilling, T.F. (2006). Cranial neural crest and development of the head skeleton. Advances in experimental medicine and biology 589, 120-133.

Kondo, S., Schutte, B.C., Richardson, R.J., Bjork, B.C., Knight, A.S., Watanabe, Y., Howard, E., de Lima, R.L., Daack-Hirsch, S., Sander, A., et al. (2002). Mutations in IRF6 cause Van der Woude and popliteal pterygium syndromes. Nature genetics 32, 285-289.

Kousa, Y.A., and Schutte, B.C. (2016). Toward an orofacial gene regulatory network.

Developmental dynamics : an official publication of the American Association of Anatomists 245, 220-232.

Kousa, Y.A., Zhu, H., Fakhouri, W.D., Lei, Y., Kinoshita, A., Roushangar, R.R., Patel, N.K., Agopian, A.J., Yang, W., Leslie, E.J., et al. (2019). The TFAP2A-IRF6-GRHL3 genetic pathway is conserved in neurulation. Human molecular genetics 28, 1726-1737.

Lee, S., Cieply, B., Yang, Y., Peart, N., Glaser, C., Chan, P., and Carstens, R.P. (2018). Esrp1Regulated Splicing of Arhgef11 Isoforms Is Required for Epithelial Tight Junction Integrity. Cell reports 25, 2417-2430 e2415.

Lee, S., Sears, M.J., Zhang, Z., Li, H., Salhab, I., Krebs, P., Xing, Y., Nah, H.D., Williams, T., and Carstens, R.P. (2020). Cleft lip and cleft palate in Esrp1 knockout mice is associated with alterations in epithelial-mesenchymal crosstalk. Development 147.

Leslie, E.J., Mancuso, J.L., Schutte, B.C., Cooper, M.E., Durda, K.M., L'Heureux, J., Zucchero, T.M., Marazita, M.L., and Murray, J.C. (2013). Search for genetic modifiers of IRF6 and genotype-phenotype correlations in Van der Woude and popliteal pterygium syndromes. American journal of medical genetics Part A 161A, 2535-2544.

Li, E.B., Truong, D., Hallett, S.A., Mukherjee, K., Schutte, B.C., and Liao, E.C. (2017). Rapid functional analysis of computationally complex rare human IRF6 gene variants using a novel zebrafish model. PLoS genetics 13, e1007009.

Li, H., Jones, K.L., Hooper, J.E., and Williams, T. (2019). The molecular anatomy of mammalian upper lip and primary palate fusion at single cell resolution. Development 146. Lieschke, G.J., and Currie, P.D. (2007). Animal models of human disease: zebrafish swim into view. Nature reviews Genetics 8, 353-367.

Liu, H., Leslie, E.J., Jia, Z., Smith, T., Eshete, M., Butali, A., Dunnwald, M., Murray, J., and Cornell, R.A. (2016). Irf6 directly regulates Klf17 in zebrafish periderm and Klf4 in murine oral epithelium, and dominant-negative KLF4 variants are present in patients with cleft lip and palate. Human molecular genetics 25, 766-776.

Liu, K., Petree, C., Requena, T., Varshney, P., and Varshney, G.K. (2019). Expanding the CRISPR Toolbox in Zebrafish for Studying Development and Disease. Frontiers in cell and developmental biology 7,13.

Losa, M., Risolino, M., Li, B., Hart, J., Quintana, L., Grishina, I., Yang, H., Choi, I.F., Lewicki, P., Khan, S., et al. (2018). Face morphogenesis is promoted by Pbx-dependent EMT via regulation of Snail1 during frontonasal prominence fusion. Development 145. Marazita, M.L. (2012). The evolution of human genetic studies of cleft lip and cleft palate. Annual review of genomics and human genetics 13, 263-283.

Meeker, N.D., Hutchinson, S.A., Ho, L., and Trede, N.S. (2007). Method for isolation of PCRready genomic DNA from zebrafish tissues. BioTechniques 43, 610, 612, 614. 
Montague, T.G., Cruz, J.M., Gagnon, J.A., Church, G.M., and Valen, E. (2014). CHOPCHOP: a CRISPR/Cas9 and TALEN web tool for genome editing. Nucleic acids research 42, W401407.

Mork, L., and Crump, G. (2015). Zebrafish Craniofacial Development: A Window into Early Patterning. Current topics in developmental biology 115, 235-269.

Motta-Mena, L.B., Reade, A., Mallory, M.J., Glantz, S., Weiner, O.D., Lynch, K.W., and Gardner, K.H. (2014). An optogenetic gene expression system with rapid activation and deactivation kinetics. Nature chemical biology 10, 196-202.

Ran, F.A., Hsu, P.D., Wright, J., Agarwala, V., Scott, D.A., and Zhang, F. (2013). Genome engineering using the CRISPR-Cas9 system. Nature protocols 8, 2281-2308.

Reid, B.S., Yang, H., Melvin, V.S., Taketo, M.M., and Williams, T. (2011). Ectodermal Wnt/beta-catenin signaling shapes the mouse face. Developmental biology 349, 261-269. Richardson, R.J., Dixon, J., Malhotra, S., Hardman, M.J., Knowles, L., Boot-Handford, R.P., Shore, P., Whitmarsh, A., and Dixon, M.J. (2006). Irf6 is a key determinant of the keratinocyte proliferation-differentiation switch. Nature genetics 38, 1329-1334. Rorick, N.K., Kinoshita, A., Weirather, J.L., Peyrard-Janvid, M., de Lima, R.L., Dunnwald, M., Shanske, A.L., Moretti-Ferreira, D., Koillinen, H., Kere, J., et al. (2011). Genomic strategy identifies a missense mutation in WD-repeat domain 65 (WDR65) in an individual with Van der Woude syndrome. American journal of medical genetics Part A 155A, 1314-1321. Sabel, J.L., d'Alencon, C., O'Brien, E.K., Van Otterloo, E., Lutz, K., Cuykendall, T.N., Schutte, B.C., Houston, D.W., and Cornell, R.A. (2009). Maternal Interferon Regulatory Factor 6 is required for the differentiation of primary superficial epithelia in Danio and Xenopus embryos. Developmental biology 325, 249-262.

Sander, J.D., Zaback, P., Joung, J.K., Voytas, D.F., and Dobbs, D. (2007). Zinc Finger Targeter (ZiFiT): an engineered zinc finger/target site design tool. Nucleic acids research 35, W599605.

Schilling, T.F., and Le Pabic, P. (2009). Fishing for the signals that pattern the face. Journal of biology $8,101$.

Swartz, M.E., Sheehan-Rooney, K., Dixon, M.J., and Eberhart, J.K. (2011). Examination of a palatogenic gene program in zebrafish. Developmental dynamics : an official publication of the American Association of Anatomists 240, 2204-2220.

Thisse, C., and Thisse, B. (2008). High-resolution in situ hybridization to whole-mount zebrafish embryos. Nature protocols 3, 59-69.

Van Otterloo, E., Williams, T., and Artinger, K.B. (2016). The old and new face of craniofacial research: How animal models inform human craniofacial genetic and clinical data.

Developmental biology 415, 171-187.

Wada, N., Javidan, Y., Nelson, S., Carney, T.J., Kelsh, R.N., and Schilling, T.F. (2005). Hedgehog signaling is required for cranial neural crest morphogenesis and chondrogenesis at the midline in the zebrafish skull. Development 132, 3977-3988.

Warzecha, C.C., Sato, T.K., Nabet, B., Hogenesch, J.B., and Carstens, R.P. (2009). ESRP1 and ESRP2 are epithelial cell-type-specific regulators of FGFR2 splicing. Molecular cell 33, 591601.

Yuan, Q., Blanton, S.H., and Hecht, J.T. (2011). Genetic causes of nonsyndromic cleft lip with or without cleft palate. Advances in oto-rhino-laryngology 70, 107-113.

Zucchero, T.M., Cooper, M.E., Maher, B.S., Daack-Hirsch, S., Nepomuceno, B., Ribeiro, L., Caprau, D., Christensen, K., Suzuki, Y., Machida, J., et al. (2004). Interferon regulatory factor 
bioRxiv preprint doi: https://doi.org/10.1101/2020.06.14.149773; this version posted June 30, 2020. The copyright holder for this preprint (which was not certified by peer review) is the author/funder. All rights reserved. No reuse allowed without permission.

6 (IRF6) gene variants and the risk of isolated cleft lip or palate. The New England journal of medicine $351,769-780$. 
Figure 1

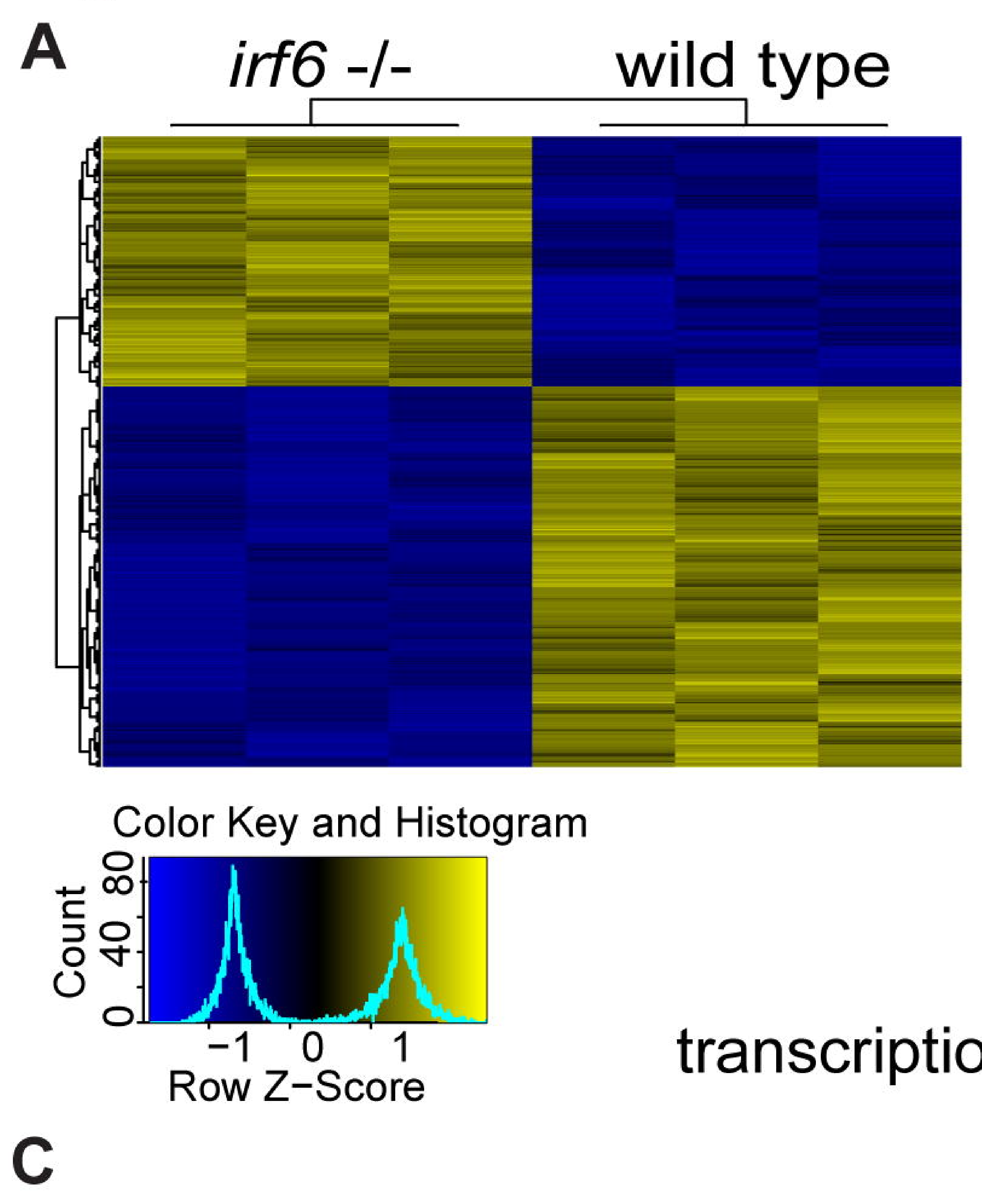

B $\quad$ NS $\quad \log 2 F C \circ P \circ P \& \log 2 F C$

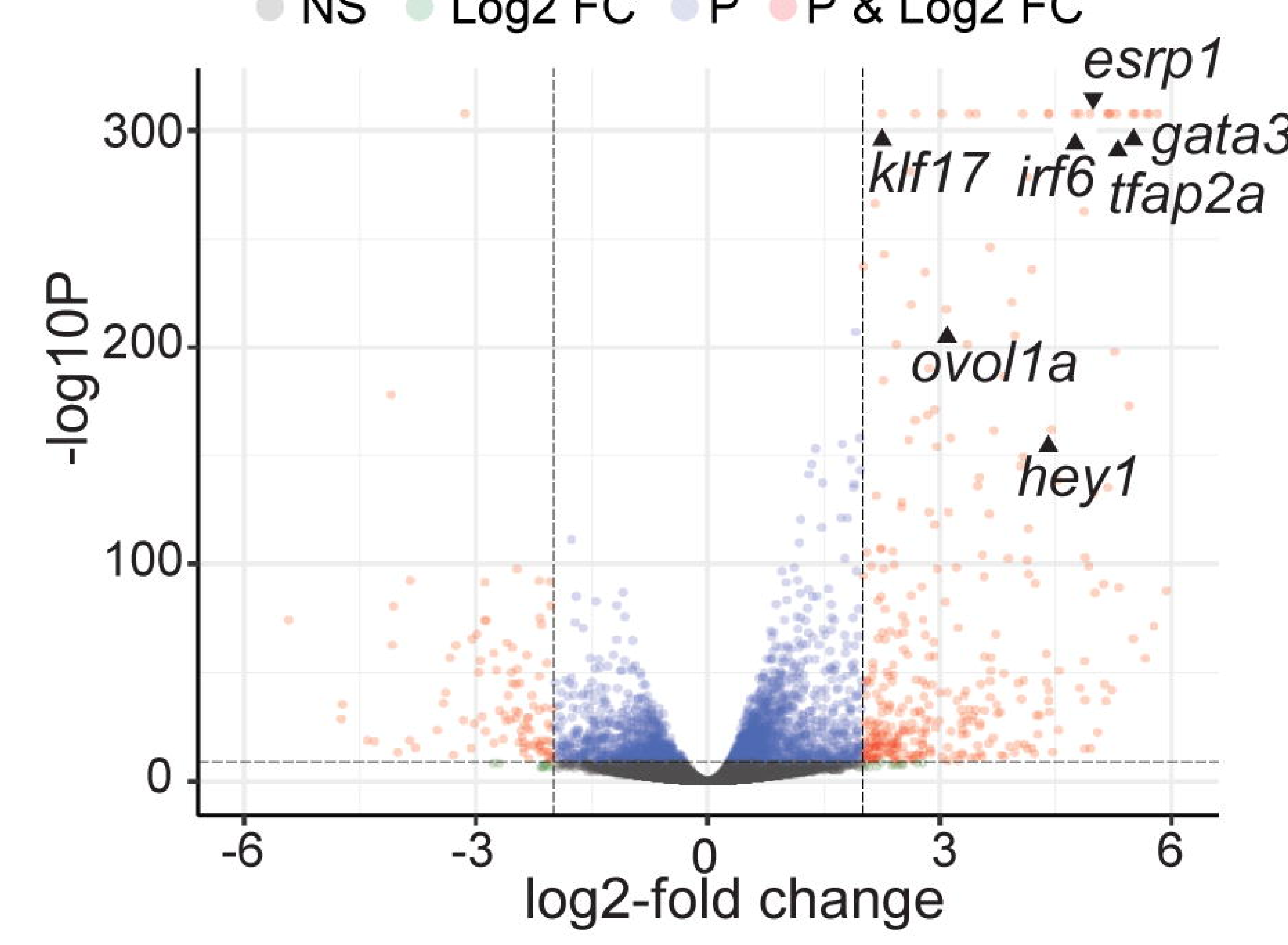

\section{tfap2a gata3}

signal receptor binding

structural molecule activity
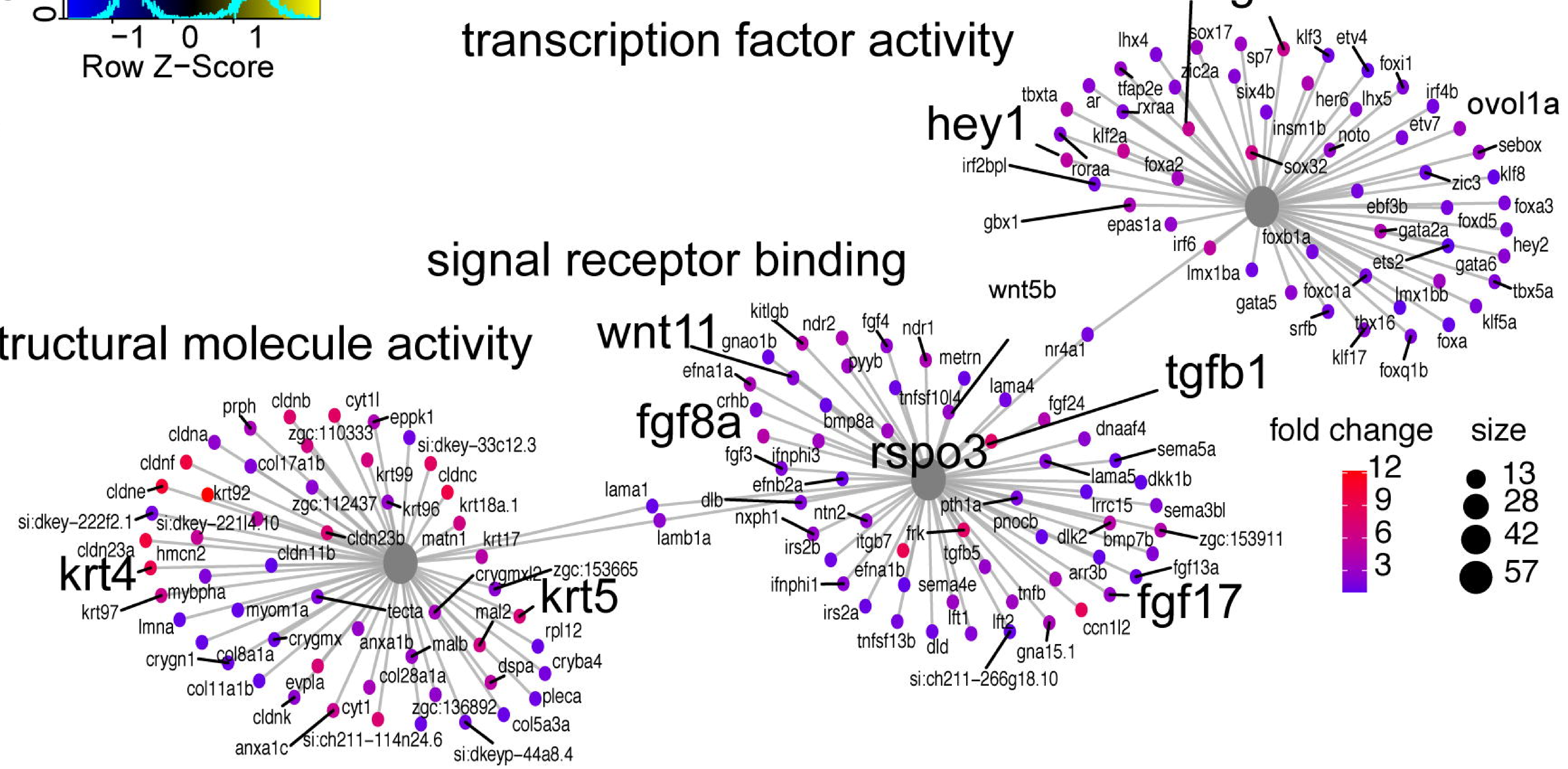

D

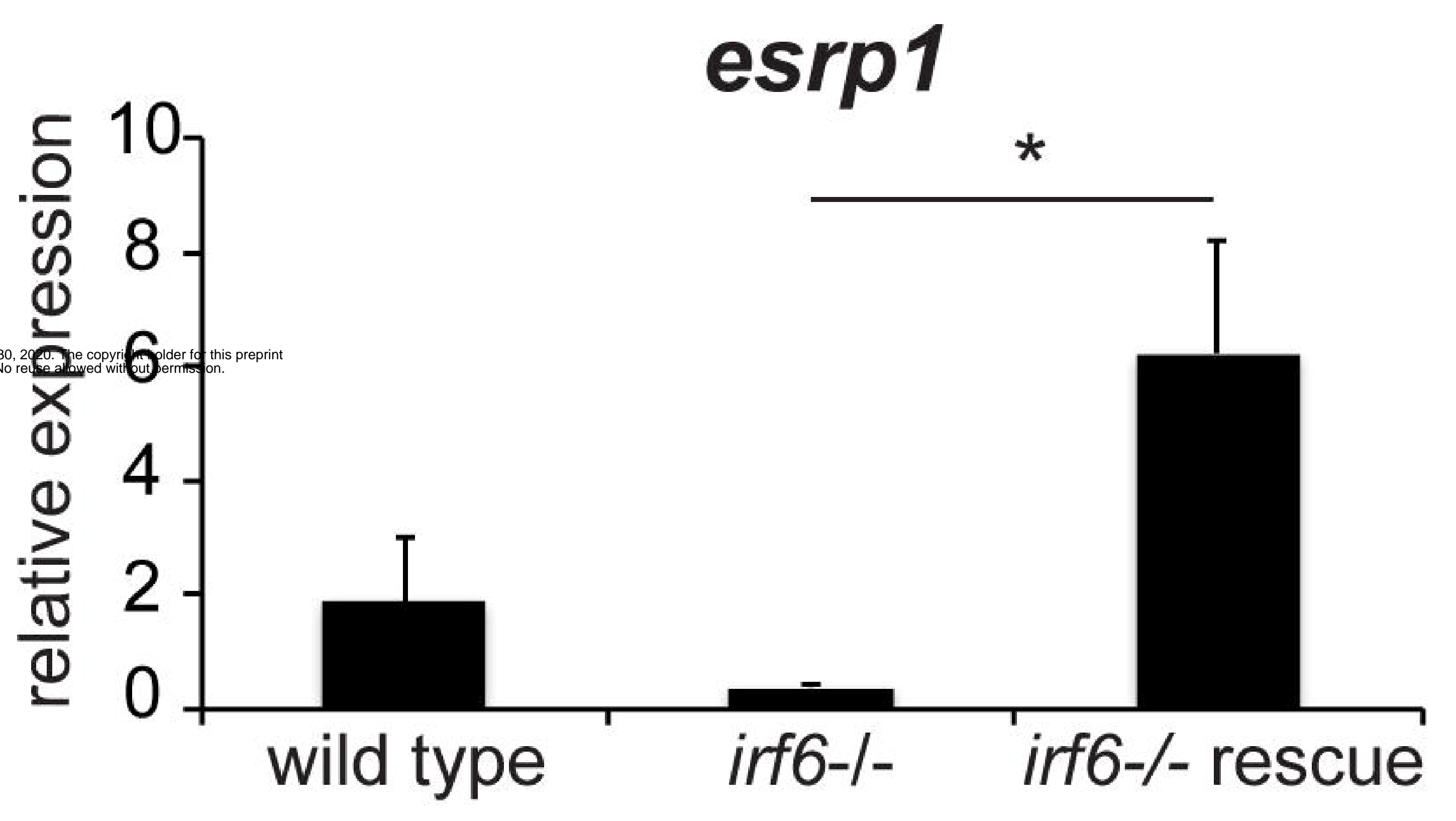


Figure 2
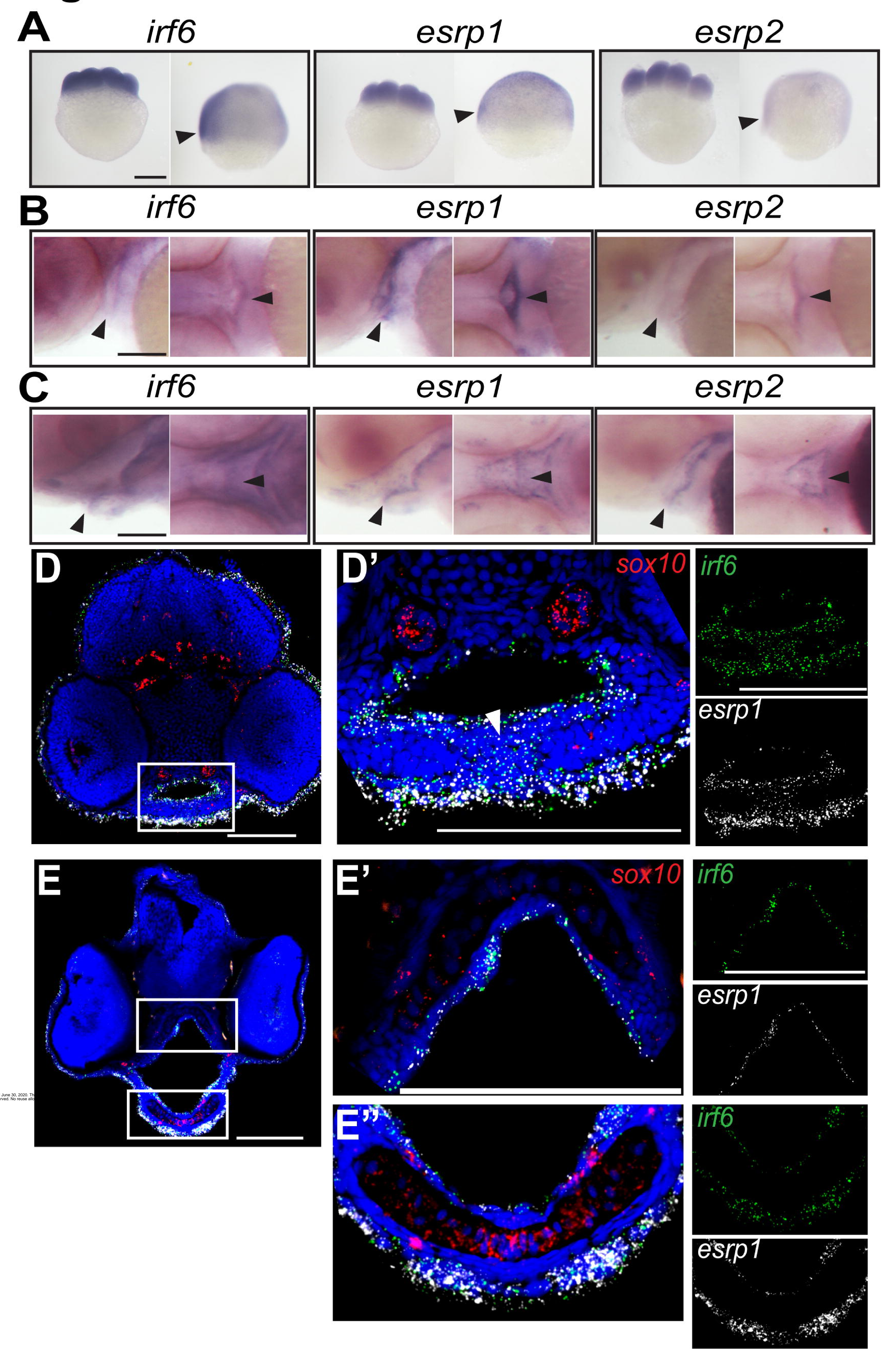
Figure 3

A

Esrp1

Esrp2
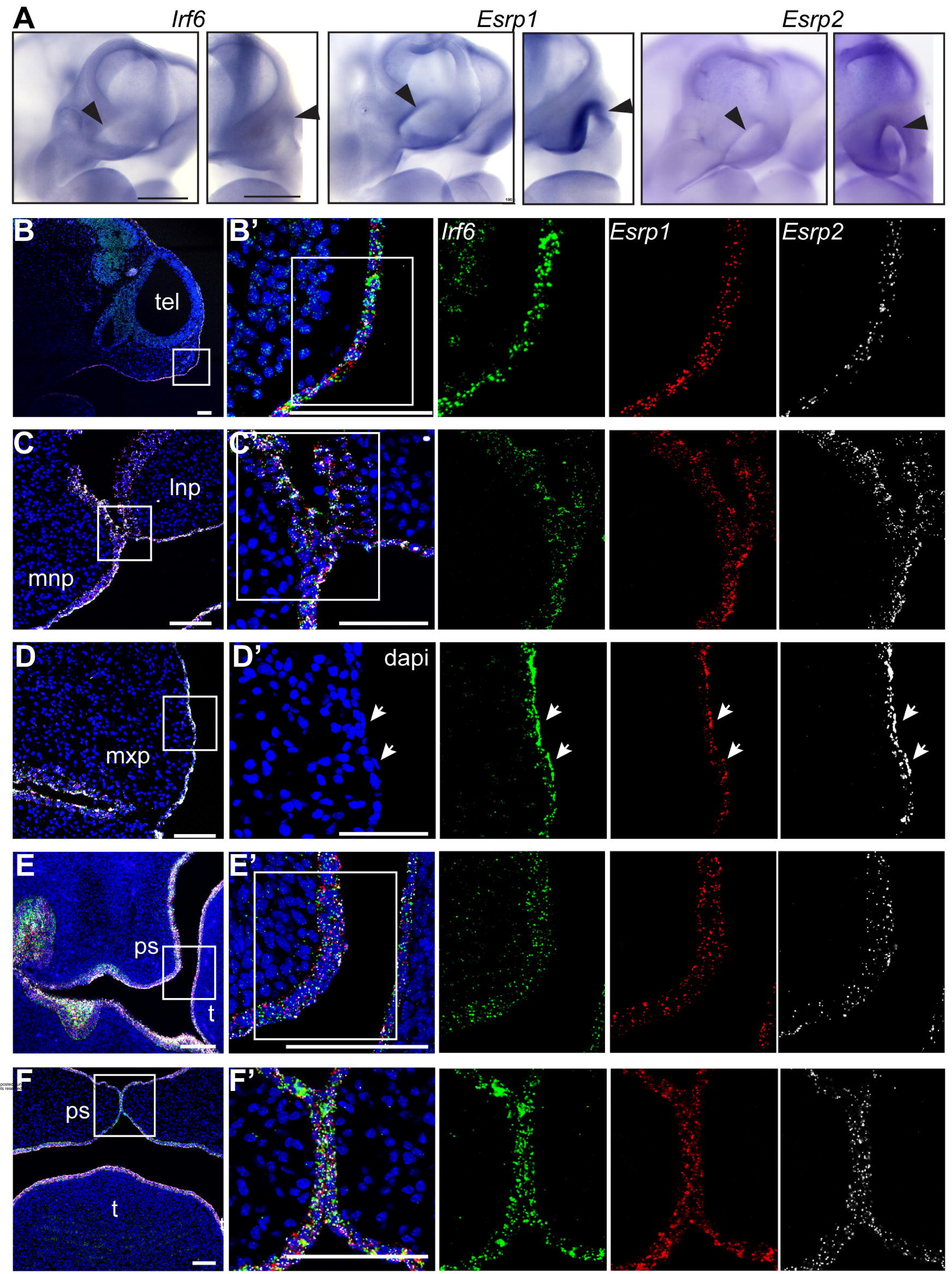
Figure 4
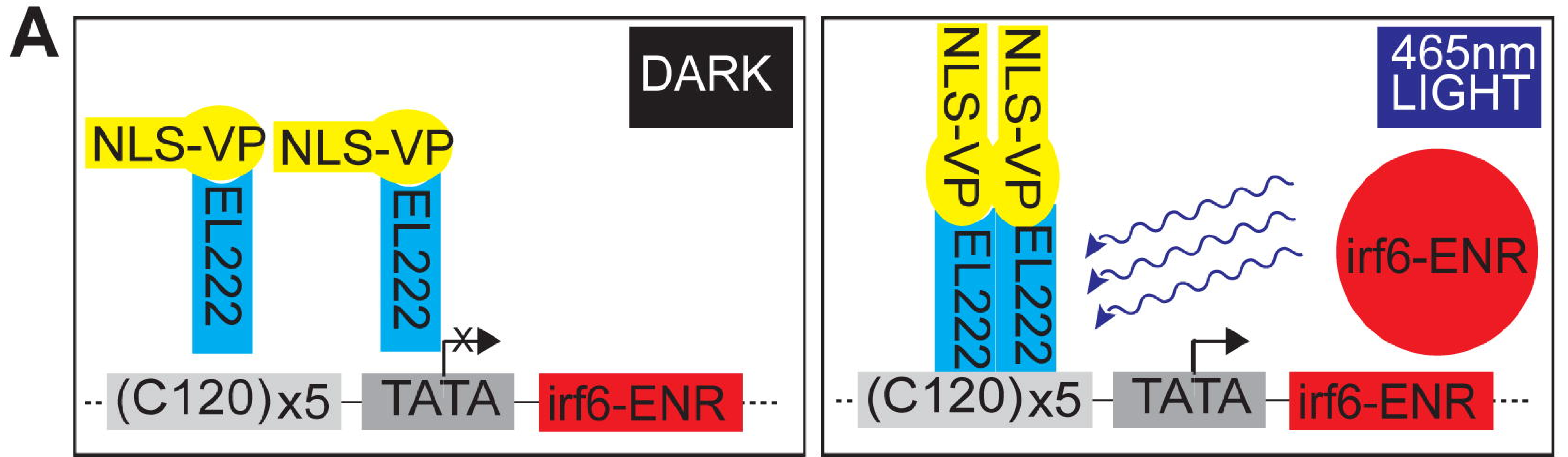

Cell Epiboly :Neural Crest Neural Crest Craniofacial ProliferationGastrulation;Specification Migration Morphogenesis
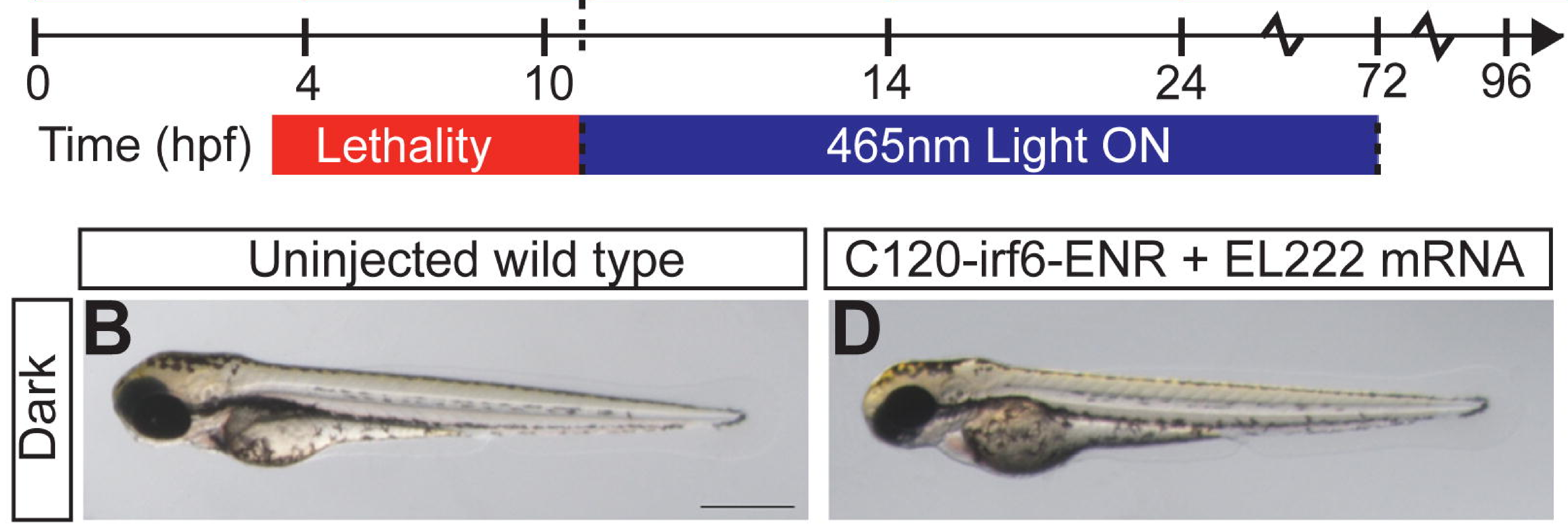

C120-irf6-ENR + EL222 mRNA
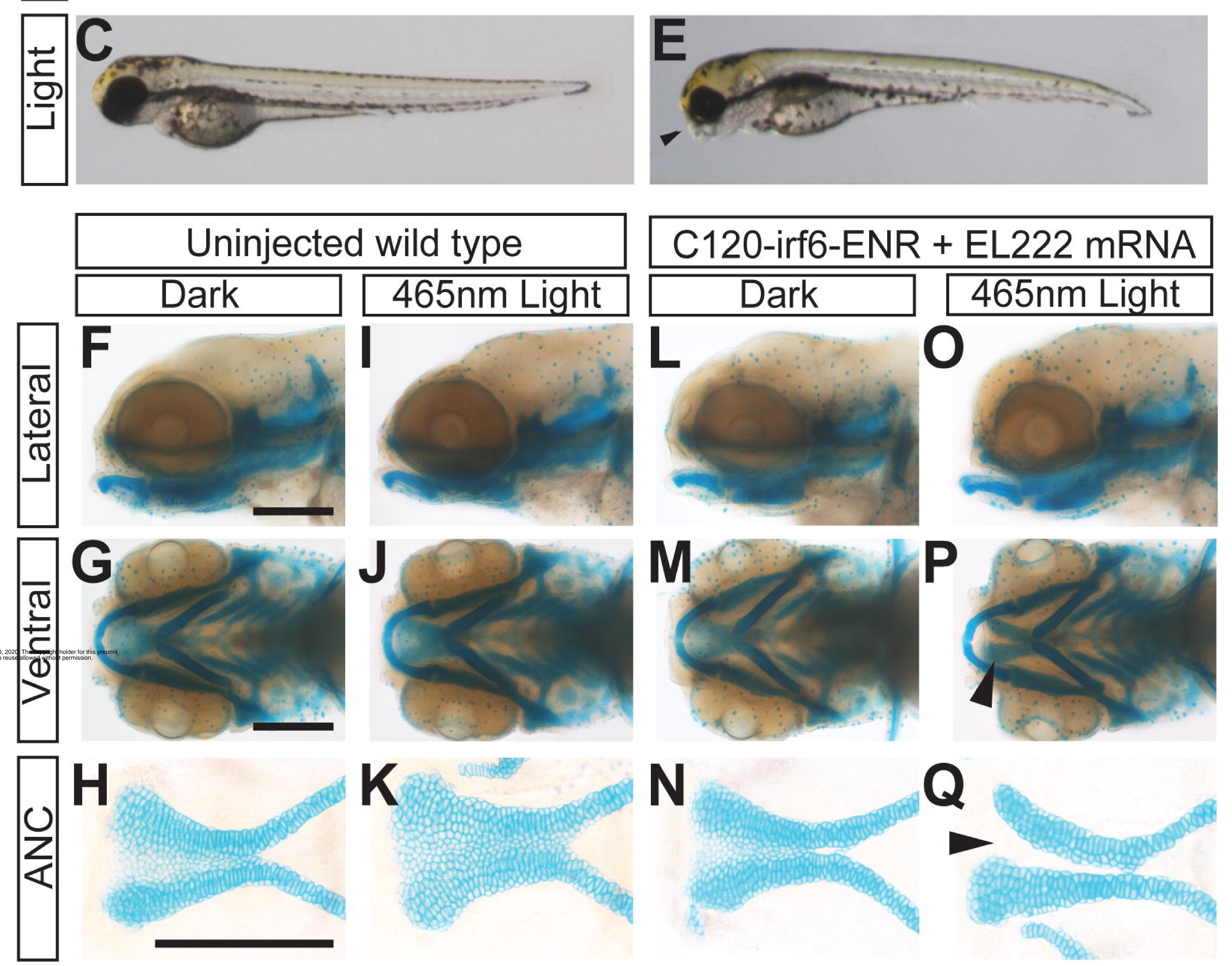

$\mathbf{N}$

Q 
Figure 5

A

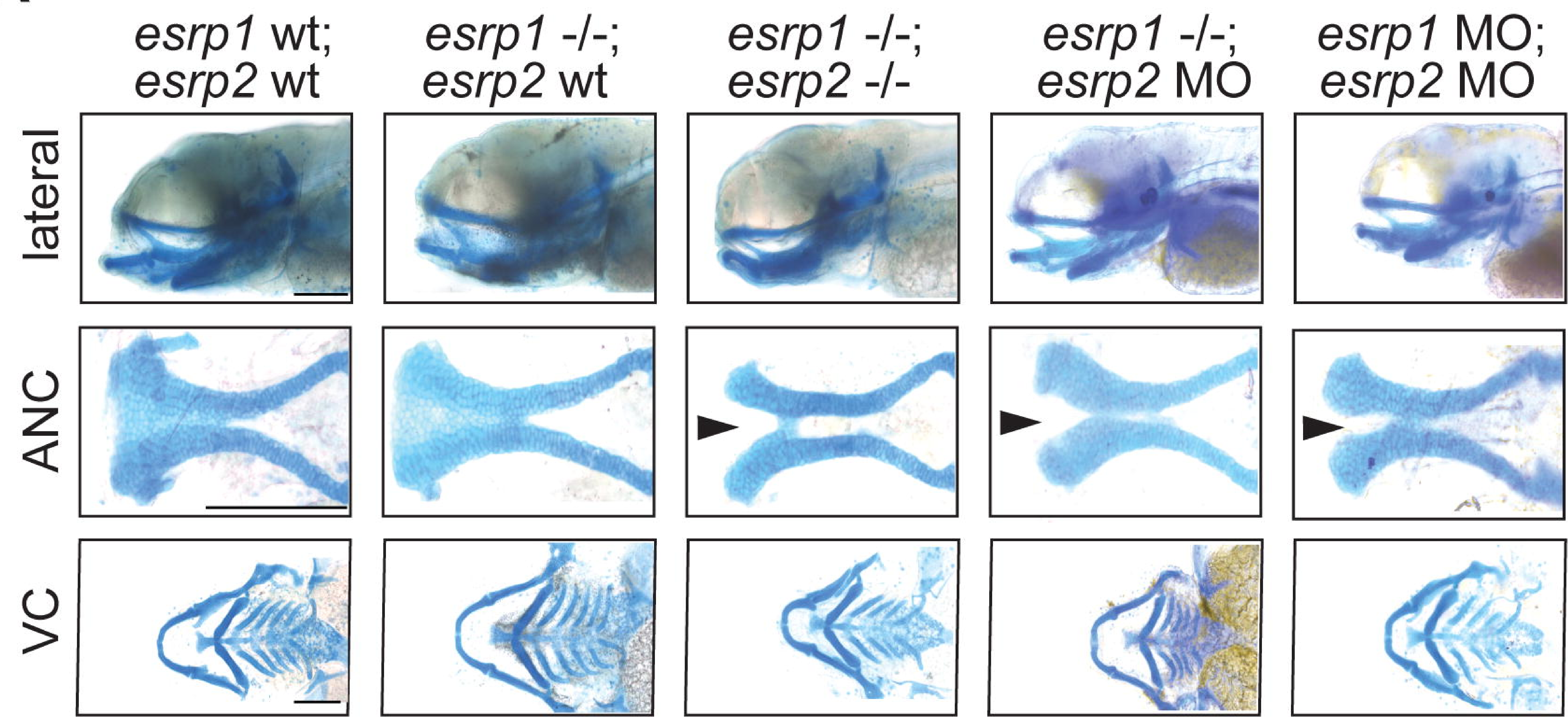

B

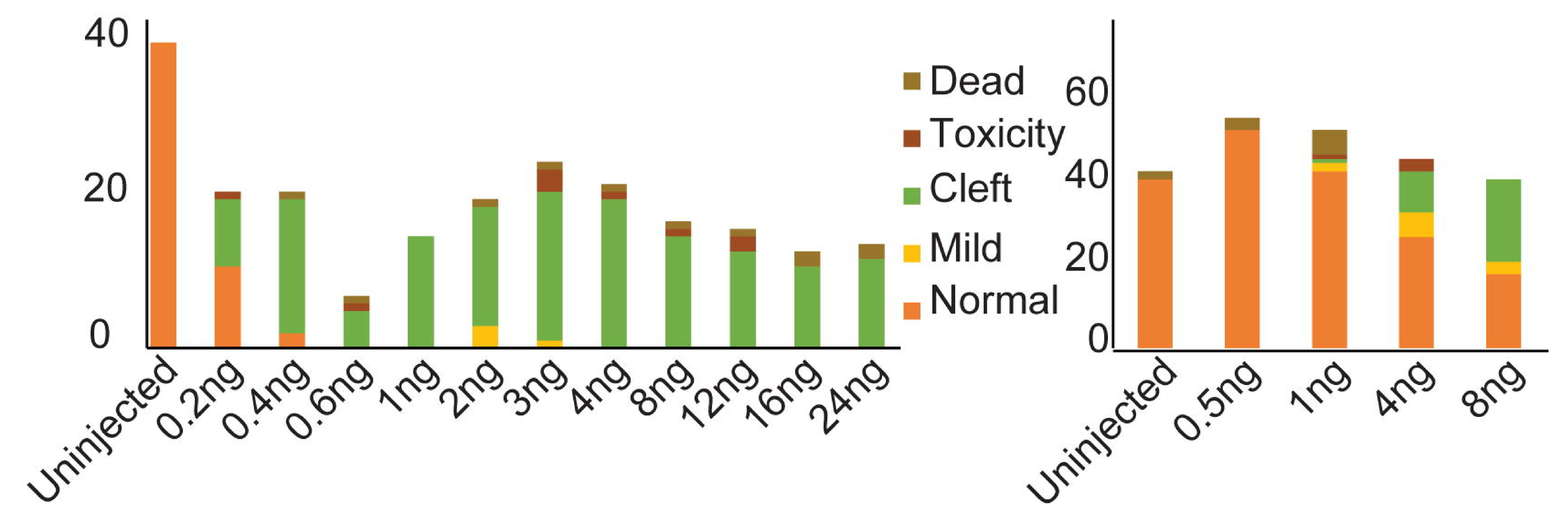

C

D
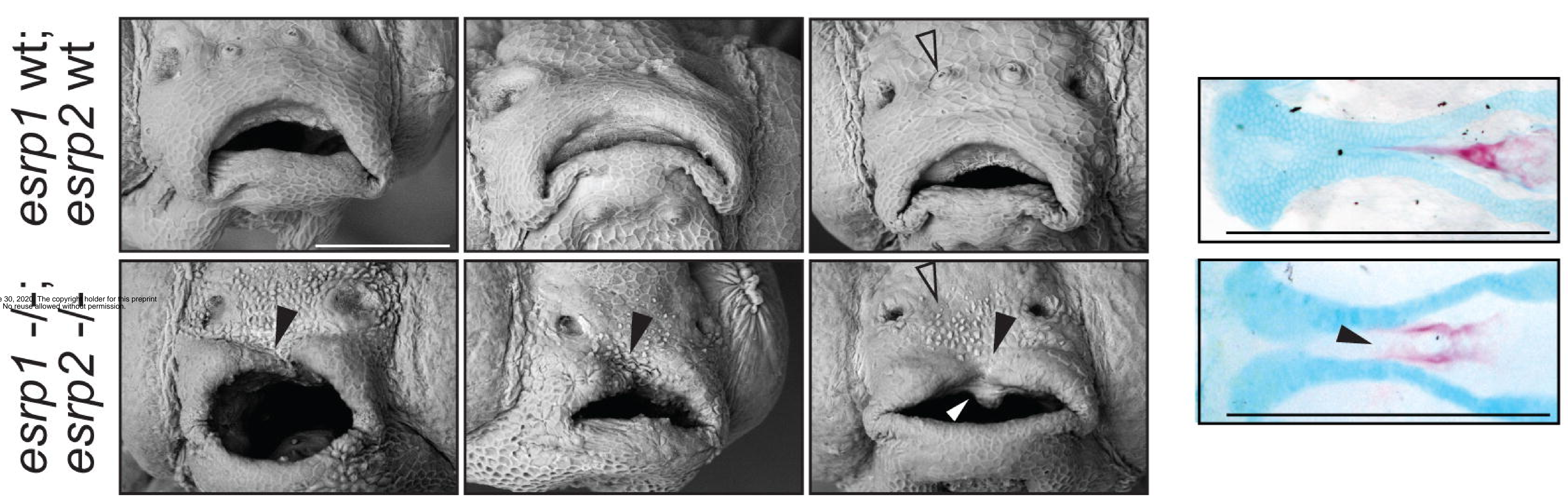


\section{Figure 6}

A wild type esrp1 -/-; esrp2 -/- esrp1 MO; esrp2
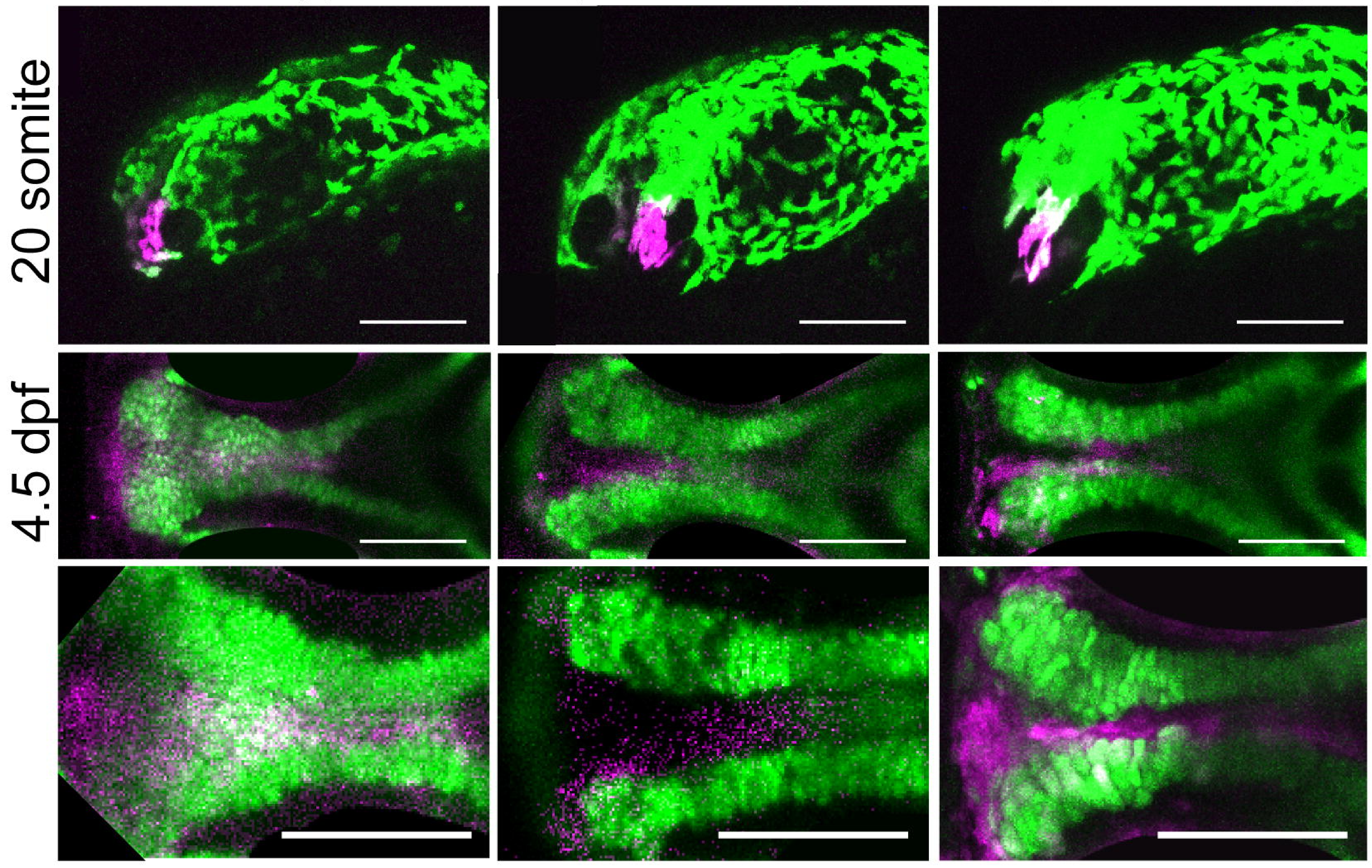

B

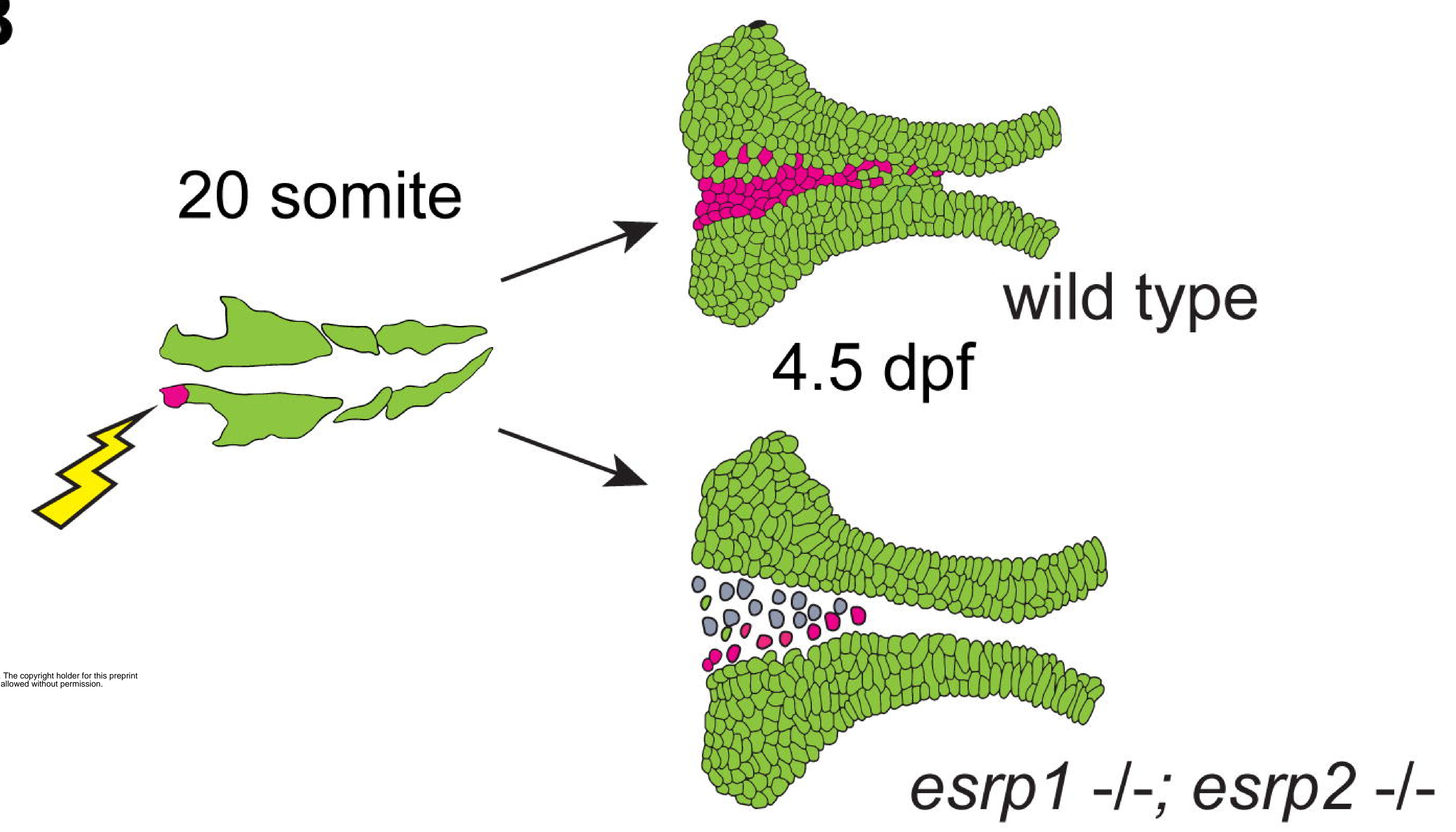


Figure 7

A

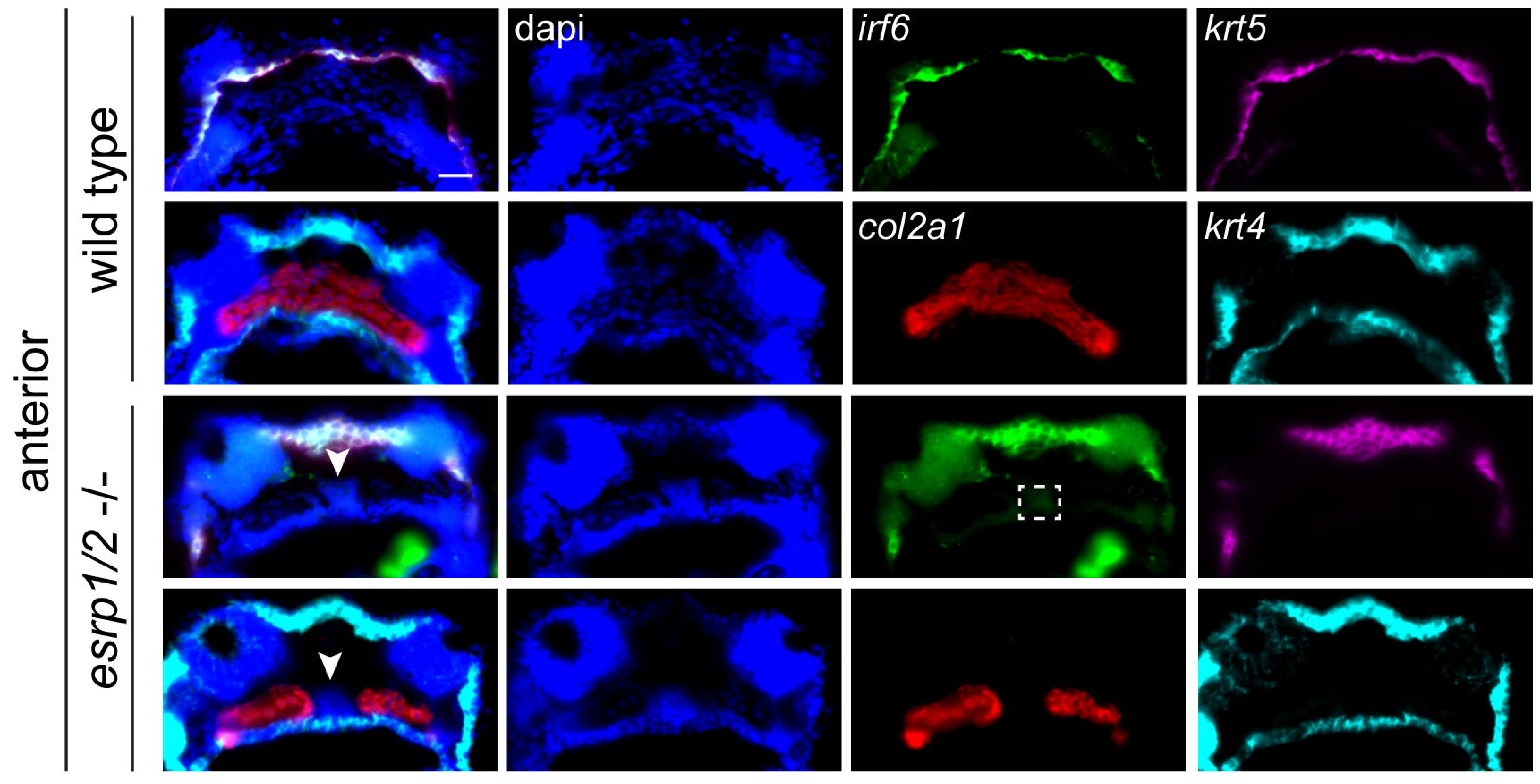

B

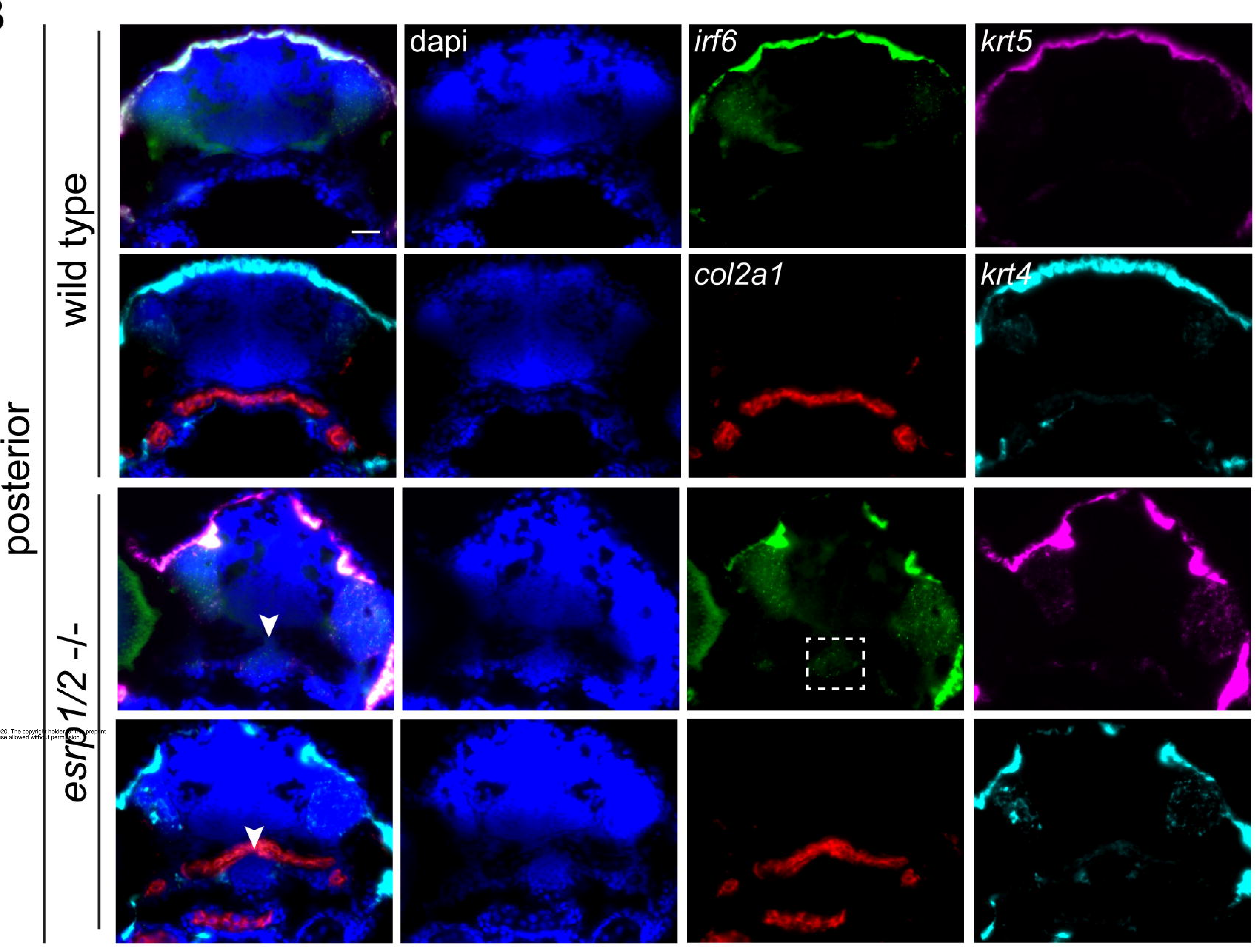


Figure 8

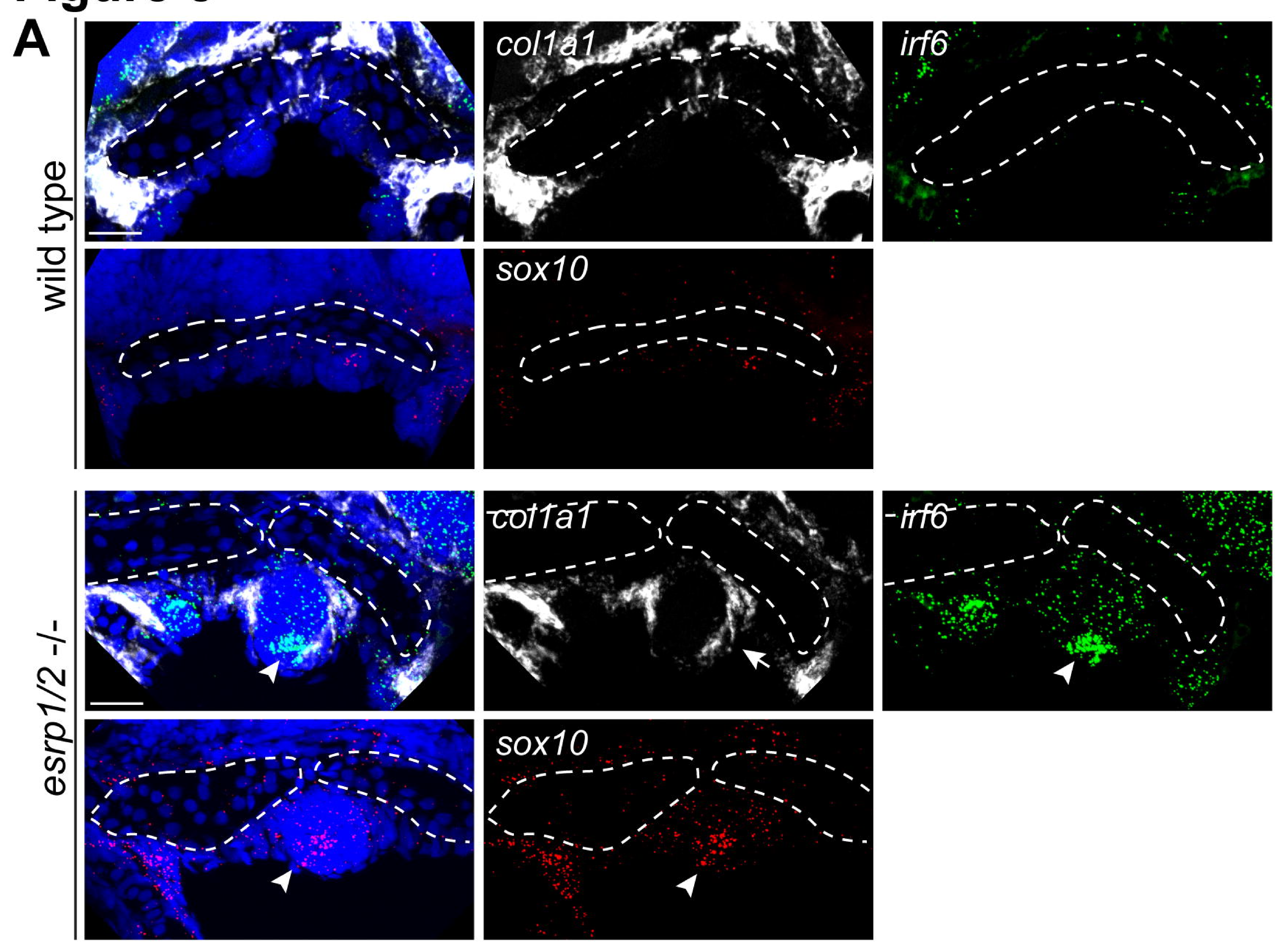

B

aberrant cells

sox10+, col1a1+, irf6+

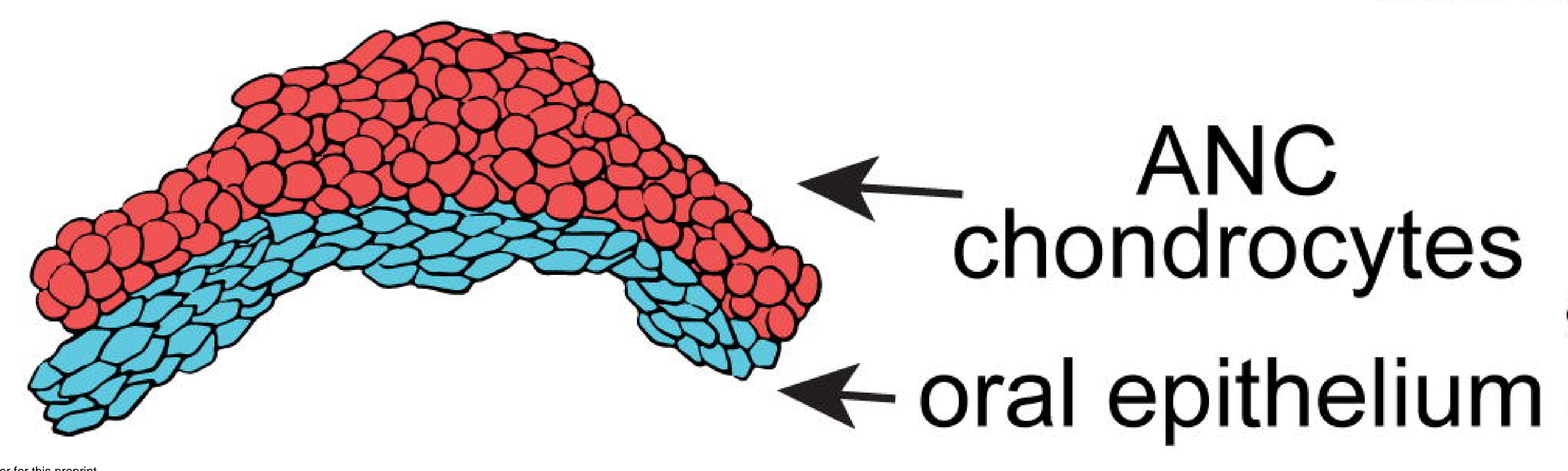

wild type
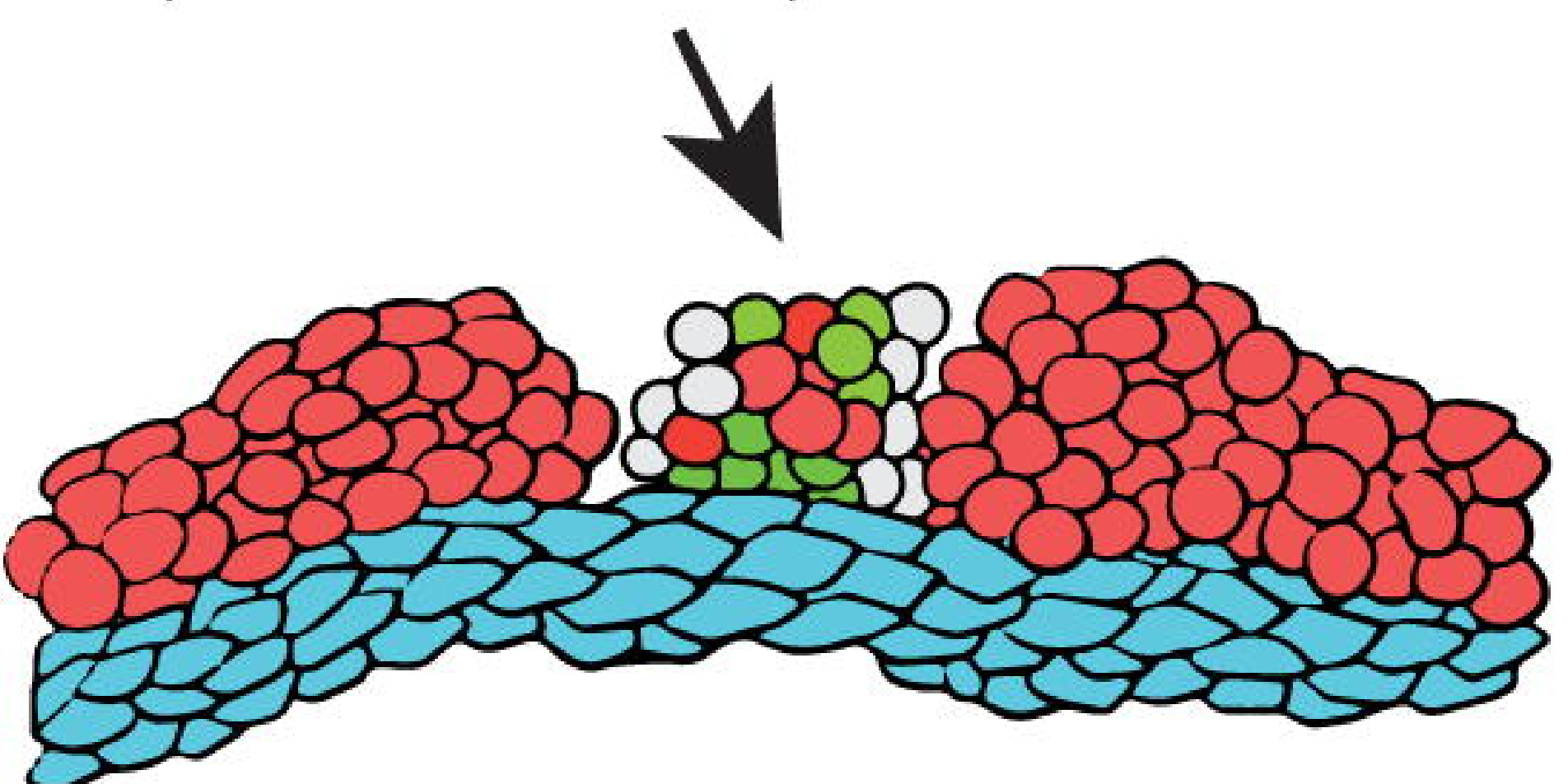

esrp1/2 -/- 
Figure 9

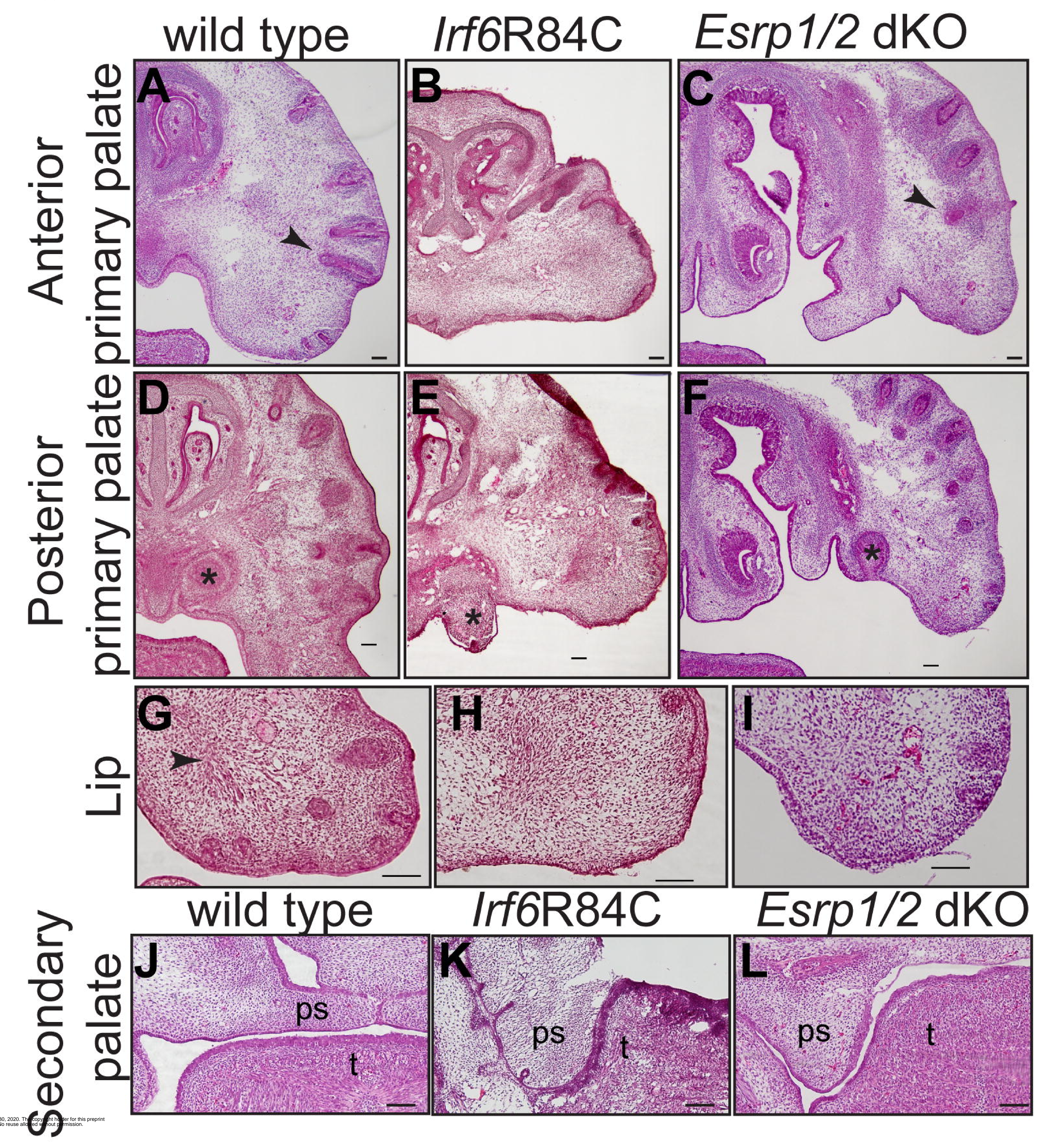

